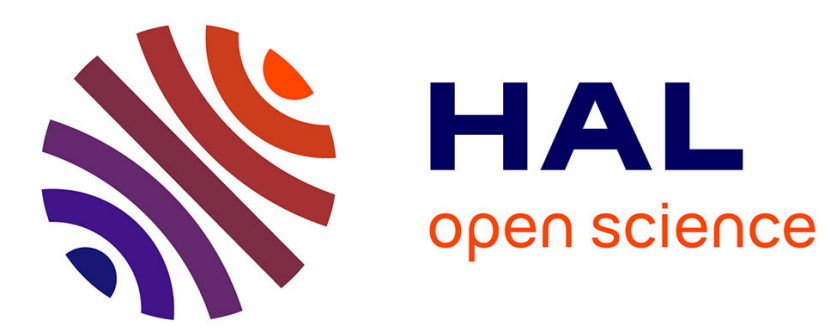

\title{
Measuring the flow and the turbulence in a harsh environment using a modified Mc Caffrey gauge
}

Christophe Proust, Didier Jamois

\section{To cite this version:}

Christophe Proust, Didier Jamois. Measuring the flow and the turbulence in a harsh environment using a modified Mc Caffrey gauge. Flow Measurement and Instrumentation, 2021, 80, pp.101993. 10.1016/j.flowmeasinst.2021.101993 . ineris-03358549

HAL Id: ineris-03358549

https://hal-ineris.archives-ouvertes.fr/ineris-03358549

Submitted on 29 Sep 2021

HAL is a multi-disciplinary open access archive for the deposit and dissemination of scientific research documents, whether they are published or not. The documents may come from teaching and research institutions in France or abroad, or from public or private research centers.
L'archive ouverte pluridisciplinaire HAL, est destinée au dépôt et à la diffusion de documents scientifiques de niveau recherche, publiés ou non, émanant des établissements d'enseignement et de recherche français ou étrangers, des laboratoires publics ou privés. 


\title{
Measuring the flow and the turbulence in a harsh environment using a modified Mc Caffrey gauge
}

\author{
Christophe Proust ${ }^{a, b}$ Didier Jamois ${ }^{a}$ \\ E-mail: christophe.proust@ineris.fr \\ ${ }^{a}$ Institut National de l'Environnement Industriel et des Risques, Parc Technologique ALATA, \\ BP 2, 60550 Verneuil-en-Halatte, France \\ ${ }^{b}$ Sorbonne Universities, UTC-TIMR, 1 rue Dr Schweitzer, Compiègne, 60200, France
}

\begin{abstract}
Considering that many laboratory measurement techniques devoted to fluid mechanics are difficult to implement in field, especially for combustion and explosions, the present authors developed during the last 2 decades, a special type of Mc Caffrey gauges to measure the flow and the turbulence in those harsh situations (figure 1). Especially a fully embedded version is now under development and the initial work about this innovation is evoked.

In this paper, the theory underpinning this technique is presented together with some technical details. Limitations are given and comparisons with reference techniques (hot wire in air jets) are presented.
\end{abstract}
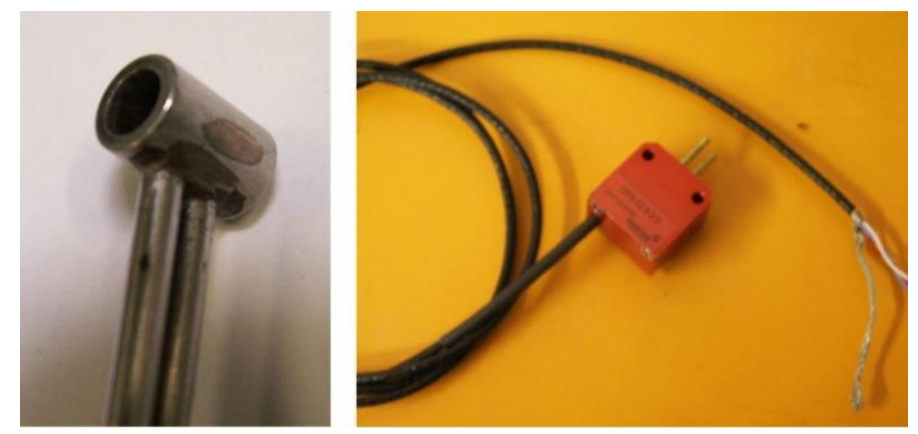

Figure 1: New Mc Caffrey device.

Keywords: flow and turbulence measurement, dust clouds, gas clouds

\section{Introduction}

In many practical situations, turbulent flows are produced which must be characterized: turbines, chemical reactors, combustion in boilers or engines... In safety too. Flow and turbulence strongly affect combustion and flame propagation. Laboratory devices like hot wires, laser Doppler techniques (LDA), Particle Image Velocimetry (PIV).... can rarely be used in field experiments. Nevertheless, estimating turbulence remains compulsory to understand the physics and model it. Some alternatives were presented in the referred paper. In the present 
paper, the authors, following the pioneering work of Tamanini (Tamanini, 1990, 1998), adapted, during the last two decades, the original Mc Caffrey technique (Mc Caffrey, 1974) to the measurement of the turbulence targeting specifically gas and dust clouds formation and turbulent flame propagation. The Mc Caffrey gauge is a 'bidirectional probe' based on the Pitot tube technique and could be used in harsh environment like fires. The paper is organized as follows. In the first section, the background of the nature of the turbulence and its characterization are recalled. A reference situation is described which is being used later, in the third section. The second section explains how the bidirectional Pitot probe can be used to measure the characteristics of the turbulence. The third section is the investigation of the performances and limitation of this technique. In particular, the influence of the design of the probe is addressed.

\section{Turbulence and associated parameters}

The reader is referred to handbooks dealing with turbulence for more detailed information (for instance Tennekes and Lumley, 1972). For the present purpose, it may be enough to recall that turbulence appears spontaneously in sheared region of flows, near the boundaries or in the wake of obstacles. The phenomenology of turbulence is intrinsically linked to the nature of the Navier-Stokes equations which are both dispersive (convective terms) and dissipative (viscous terms). Where the former dominates, a perturbation grows, distorts and is shattered into smaller structures. Where the later dominate, perturbations are dampened and transformed into heat. A typical example is the boundary near the wall in a duct or past a sphere as shown in Figure 2left. A small instability appears in the boundary flow, develops as an eddy, and breaks down in a variety of smaller and smaller flow structures down to the scale where viscous forces dissipate them. From a measurement point of view, a point velocity measurement might exhibit the form shown in Figure 2-right with a mean velocity carrying random fluctuations. The random fluctuations are produced by the turbulence structures conveyed by the mean flow. The chaotic behaviour comes from the existence of many flow structures of variable scales called the "turbulent cascade".
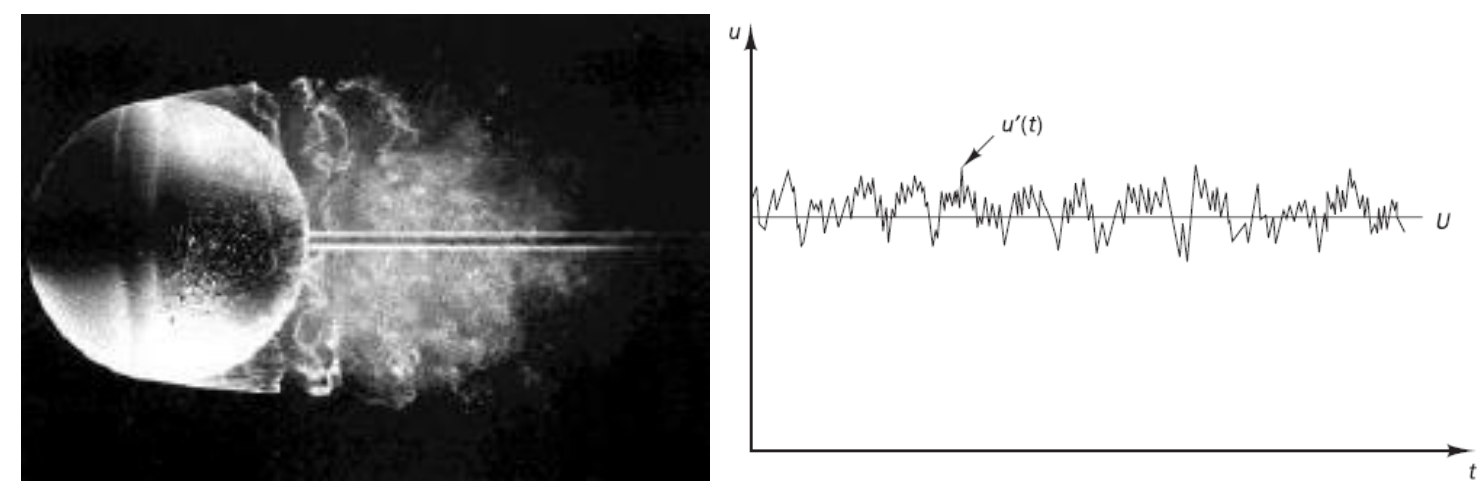

\section{Figure 2: development of turbulence in a flow past a sphere (left) and typical velocity recording in a turbulent flow (right)}

Many aspects of the turbulence have been clarified since the pioneering work of Reynolds. For the purpose of giving the definitions and parameters used later, some aspects are recalled. A very usual description means is to decompose the instantaneous velocity $u(t)$ into a steady average value $U$ and a randomly fluctuating (turbulent) component $\mathrm{u}^{\prime}(\mathrm{t})$ so that $\mathrm{u}(\mathrm{t})=\mathrm{U}+$ $\mathrm{u}^{\prime}(\mathrm{t})$. A turbulent flow is then analysed in terms of an interaction between the averaged values of flow properties $(\mathrm{U}, \mathrm{V}, \mathrm{W}, \mathrm{P} \ldots)$ with some statistical properties of their fluctuations (rms of $\left.\mathrm{u}^{\prime}(t), \mathrm{v}^{\prime}(t), \mathrm{w}^{\prime}(t), \mathrm{p}^{\prime}(\mathrm{t}) \ldots\right)$ produced by the passage of the various eddies on the measuring point. The intensity of the turbulence is the root mean square of the velocity 
fluctuations (called $u^{\prime}$ for the rms of $\mathrm{u}^{\prime}(\mathrm{t})$ in the following). It was shown that the turbulent cascade can be described by the rms of the velocity fluctuations, $u$ ', and by the integral scale of the turbulence, $\mathrm{L}_{\mathrm{int}}$, which is a measure of the scale of the largest eddies of the flow carrying most of the energy of the turbulent part of the flow. In practice:

$$
R(x)=\frac{1}{x_{\text {meas }}} \cdot \int_{0}^{x_{\text {meas }}} \frac{u\left(x^{\prime}\right) \cdot u\left(x^{\prime}-x\right)}{u^{\prime 2}} \cdot d x^{\prime} \quad[1] \quad \text { and } \quad L_{\text {int }}=\int_{0}^{+\infty} R(x) \cdot d x \quad \text { [2] }
$$

Where $\mathrm{x}_{\text {meas }}$ is large as compared to $\mathrm{L}_{\mathrm{int}}$ ( $\mathrm{x}_{\text {meas }}$ tend to infinity). $\mathrm{R}(\mathrm{x})$ is the correlation coefficient between the instantaneous velocity measured at two point separated by a distance x. Obviously when $\mathrm{x}=0, \mathrm{R}(\mathrm{x}=0)=1$. When $\mathrm{x}$ is larger than the largest eddies, the information from the turbulent cascade is lost and $\mathrm{R}$ drops to zero.

The "spectral energy" $\mathrm{E}(\mathrm{\kappa})$ describes the turbulent cascade of a flow based on the former parameters u' and $\mathrm{L}_{\text {int. }}$. The spectral energy $\mathrm{E}(\mathrm{\kappa})$ (units $\mathrm{m}^{3} / \mathrm{s}^{2}$ ) is the kinetic energy per unit mass and per unit wavenumber of fluctuations around the wavenumber $\kappa(\kappa=2 \pi / \lambda$, where $\lambda$ is a given wavelength/size of the eddies):

$$
E(\kappa)=\frac{2}{\pi} \cdot u^{\prime 2} \cdot \int_{0}^{\infty} R(x) \cdot \cos (\kappa \cdot x) \cdot d x
$$

For high Reynolds flows ${ }^{1}$, it can be shown (Hinze, 1975) that $R(x)=\exp \left(-x / L_{\text {int }}\right)$ and a simple expression for $\mathrm{E}(\kappa)$ is obtained:

$$
E(\kappa)=\frac{2}{\pi} \cdot u^{\prime 2} \cdot L_{i n t} \cdot \frac{1}{1+\kappa^{2} \cdot L_{\text {int }}^{2}}
$$

The ratio $\frac{\mathrm{E}(\kappa)}{u^{2} \cdot L}=f\left(\kappa \cdot L_{\text {int }}\right)$ is a sort of "universal" non-dimensional turbulence spectrum for high Reynolds flows (Figure 3).

In many studies dedicated to turbulence measurement, the "grid" turbulence spectrum is often used as a reference situation. However, the turbulence intensity is rather small so as the scales breadth. This is rather far from the practical situations envisioned in this paper.

Another reference situation was thus chosen: the subsonic round free jet. A large body of knowledge was accumulated since the middle of the $20^{\text {th }}$ century, both theoretically and experimentally (Wygnanski, 1969; Birch, 1983; Moodie, 1990; Djeridane, 1994; Carazzo, 2006; Ball, 2012). Only the salient points are recalled here. The jet is a cone with an opening angle on the order of $24^{\circ}$ corresponding to the natural development of a free boundary layer, thus independent from the nature of the jet. In a free jet, mass and momentum are conserved. This is a "self-similar" flow. For a subsonic jet developing in a surrounding quiet fluid with the same density, the following correlation were established (Hinze, 1975; Talbot, 2009):

$$
\frac{U_{x}}{U_{0}}=5.8 \cdot \frac{D_{0}}{x} \quad[5] \quad u^{\prime} \approx 0.26 \cdot U_{x}[6] \quad L_{\text {int }} \approx 0.05 \cdot x
$$

Where $\mathrm{U}_{0}$ and $\mathrm{U}_{\mathrm{x}}$ are respectively the average flow velocities at the orifice and on the axis of the jet at distance $\mathrm{x}$ from the orifice.

\footnotetext{
${ }^{1}$ Often true for field experiments
} 


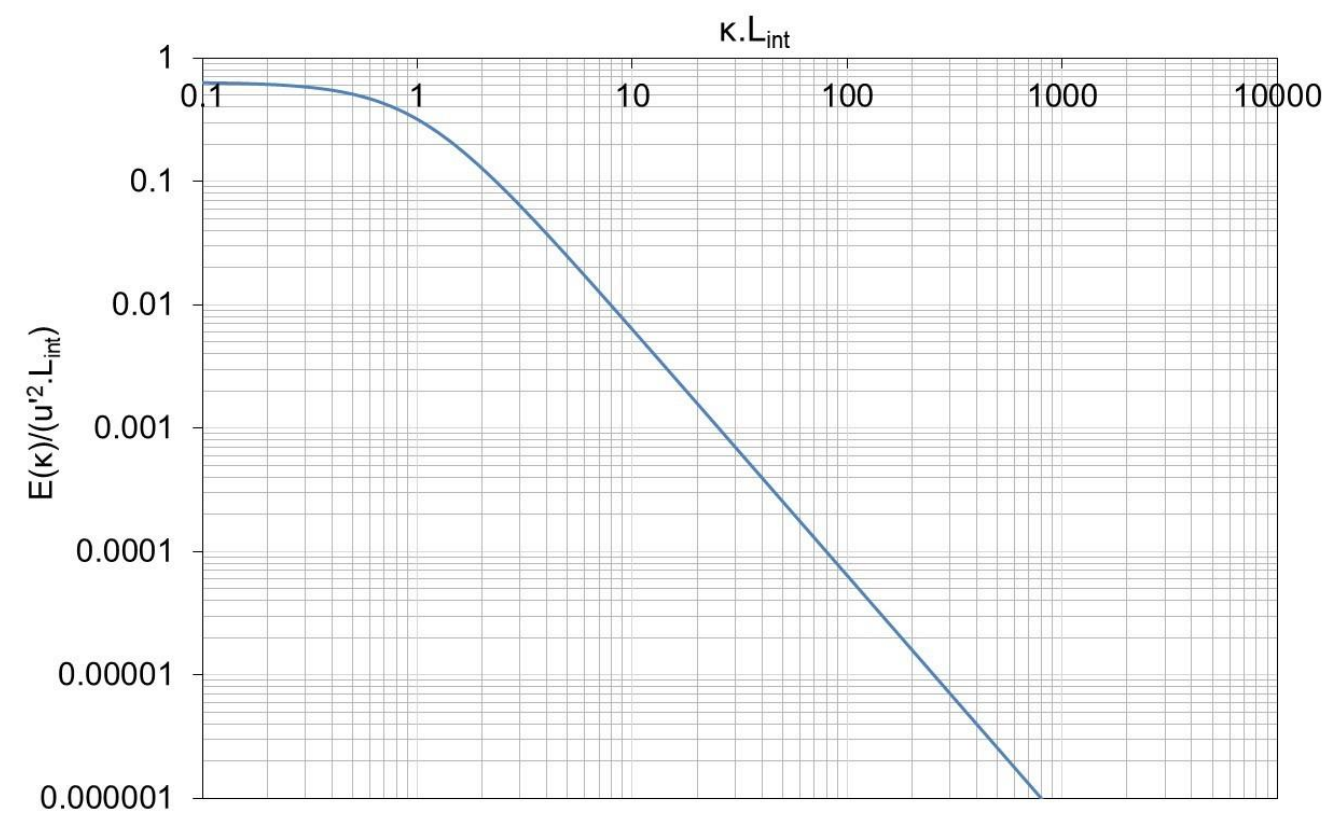

Figure 3: universal turbulence spectrum

\section{Bidirectional Pitot probes theory: flow and turbulence}

The principle of the Pitot technique is to measure the instantaneous dynamic pressure of the flow and to extract the instantaneous velocity (Jezdinsky, 1966; Becker, 1973; Kent, 1987; Cortese, 1992; Sette, 2006). The bidirectional probe, as used by Mc Caffrey, is a Pitot technique (figure 4). As shown in appendix $\mathrm{A}$, the differential pressure $\Delta \mathrm{P}$ applied by the flow on the two sides of the wall located in the middle of the head of the bidirectional probe is close to the dynamic pressure of the flow provided the angle $\theta$ of the direction of the mean flow with the axis of the probe (figure 4 ) is within $\pm 40^{\circ}$.

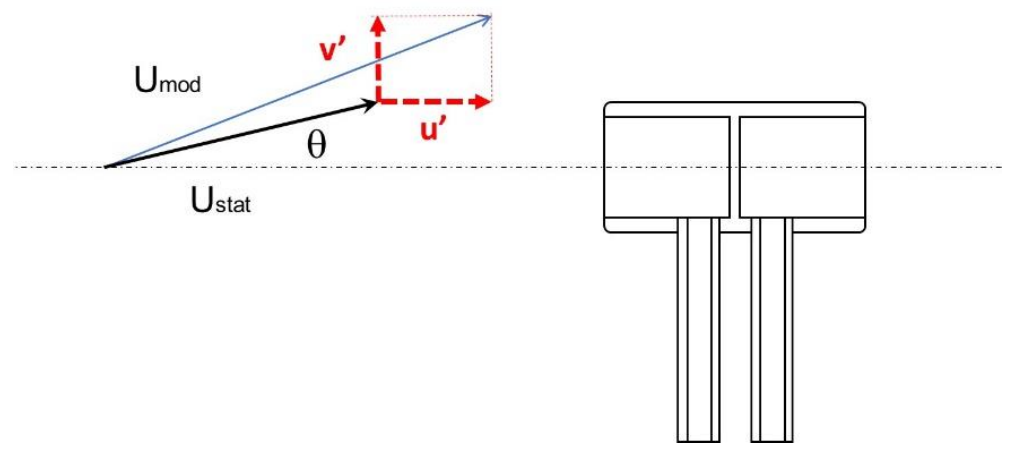

Figure 4: bidirectional probe sketch and definitions

In the following, it is assumed $-40^{\circ}<\theta<+40^{\circ}$ so that $\Delta P \approx \frac{1}{2} \cdot \rho \cdot U_{\text {mod }}^{2}$. On figure $4, \mathrm{U}_{\text {mod }}$ is the instantaneous modulus of the velocity vector and $U_{\text {stat }}$ is the average of the velocity field as defined in the Reynolds approximation. Those velocities are linked to the velocity fluctuations, u' and v' as follows:

$$
\Delta P \approx \frac{1}{2} \cdot \rho \cdot U_{\text {mod }}^{2}(t)=>\Delta P=\frac{1}{2} \cdot \rho \cdot\left[\left(U_{\text {stat }} \cdot \cos \theta+u^{\prime}(t)\right)^{2}+\left(U_{\text {stat }} \cdot \sin \theta+v^{\prime}(t)\right)^{2}\right]
$$


and:

$$
U_{\text {mod }}(t)=U_{\text {stat }} \cdot \sqrt{1+\left(\frac{u^{\prime}(t)}{U_{\text {stat }}}\right)^{2}+\left(\frac{v^{\prime}(t)}{U_{\text {stat }}}\right)^{2}+2 \cdot\left(\frac{u^{\prime}(t)}{U_{\text {stat }}} \cdot \cos \theta+\frac{v^{\prime}(t)}{U_{\text {stat }}} \cdot \sin \theta\right)}
$$

For "small enough" values of the fluctuations as compared to the averages (discussed hereafter), a first order Taylor expansion of [8] can be used giving:

$$
U_{\text {mod }}(t) \approx U_{\text {stat }} \cdot\left[1+\frac{1}{2} \cdot\left(\left(\frac{u^{\prime}(t)}{U_{\text {stat }}}\right)^{2}+\left(\frac{v^{\prime}(t)}{U_{\text {stat }}}\right)^{2}\right)+\left(\frac{u^{\prime}(t)}{U_{\text {stat }}} \cdot \cos \theta+\frac{v^{\prime}(t)}{U_{\text {stat }}} \cdot \sin \theta\right)\right]
$$

The average and fluctuations of $U_{\text {mod }}$ which are extracted from the signal, read:

$$
\begin{gathered}
\bar{U}_{\text {mod }} \approx U_{\text {stat }} \cdot\left[1+\frac{1}{2} \cdot\left(\frac{u^{\prime}}{U_{\text {stat }}}\right)^{2}+\frac{1}{2} \cdot\left(\frac{v^{\prime}}{U_{\text {stat }}}\right)^{2}\right] \\
u_{\text {mod }}^{\prime}(t)=U_{\text {mod }}(t)-\bar{U}_{\text {mod }} \\
\approx\left[\frac{1}{2} \cdot\left(\frac{u^{\prime}(t)}{U_{\text {stat }}}\right)^{2}-\frac{1}{2} \cdot\left(\frac{u^{\prime}}{U_{\text {stat }}}\right)^{2}+\frac{1}{2} \cdot\left(\frac{v^{\prime}(t)}{U_{\text {stat }}}\right)^{2}-\frac{1}{2} \cdot\left(\frac{v^{\prime}}{U_{\text {stat }}}\right)^{2}+\left(\frac{u^{\prime}(t)}{U_{\text {stat }}} \cdot \cos \theta+\frac{v^{\prime}(t)}{U_{\text {stat }}} \cdot \sin \theta\right)\right]
\end{gathered}
$$

In jets, $u^{\prime}(t)$ and $v^{\prime}(t)$ fluctuate with a typical velocity of $0.25 \mathrm{xU}_{\text {stat }}$, so that the linear terms are on the order of 0.25 whereas the quadratic terms are on the order of 0.06 . So, even in free jets, a reasonable approximation of $\bar{U}_{\text {mod }}$ and $u_{\text {mod }}^{\prime}(t)$ are:

$$
\begin{gathered}
\bar{U}_{\text {mod }} \approx U_{\text {stat }} \\
u_{\text {mod }}^{\prime}(t) \approx u^{\prime}(t) \cdot \cos \theta+v^{\prime}(t) \cdot \sin \theta
\end{gathered}
$$

It can be calculated that the rms of $u_{\text {mod }}^{\prime}(t)$ reads:

$$
u_{\text {mod }}^{\prime} \approx \sqrt{u^{\prime 2} \cdot \cos ^{2} \theta+v^{\prime 2} \cdot \sin ^{2} \theta+\overline{u^{\prime} \cdot v^{\prime}} \cdot \sin \theta \cdot \cos \theta}
$$

In a free jet, $\frac{\overline{u^{\prime} \cdot v^{\prime \prime}}}{u^{\prime 2}}$ may amount about -0.25 (Hinze, 1975) and $u^{\prime} \approx v^{\prime}$ so that depending on $\theta$, $0.93 \cdot u^{\prime} \leq u_{\text {mod }}^{\prime} \leq u^{\prime}$.

Hence, $\bar{U}_{\text {mod }}$ is indeed very close to $\mathrm{U}_{\text {stat. and }} \mathrm{u}^{\prime}$ mod is close to $\mathrm{u}^{\prime}$ (provided the probe is reasonably aligned with the flow as stated in the beginning of this section). Note that $U_{\text {mod }}$ is a good estimate of the mean flow, but $u$ 'mod is a composition of $u^{\prime}$ and $v$ '. The estimate of $u$ ' is good if $u^{\prime}$ and $v^{\prime}$ are similar. If for instance $u^{\prime}$ is twice v': $0.8 \cdot u^{\prime} \leq u_{\text {mod }}^{\prime} \leq u^{\prime}$. Nevertheless, this development shows that the bidirectional probe is capable of providing a rather accurate estimate of the turbulence of the flow along its axis.

Experiments show that other measuring aspects need to be addressed such as the influence of the size of the head and of the length of the connecting pipes on the measured turbulence. Resonance/damping effects of the head of the sensor need to be accounted for.

\section{Experimental setup}

A cylinder is used as a stagnation chamber (figure 5) and is maintained under a regulated pressure of air (usually below $650 \mathrm{mbar}$ ). A $5 \mathrm{~mm}$ "thin wall" orifice is drilled on the flange opposite to the air feed, creating a free jet of air. In this configuration a "top hat" flow field is 
created at the orifice with a discharge coefficient, $\mathrm{C}_{\mathrm{D}}$, of 0.72 (measured). In this situation,

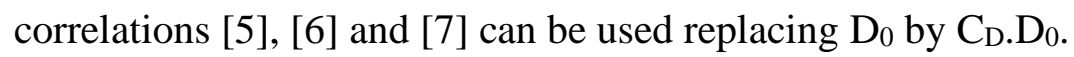

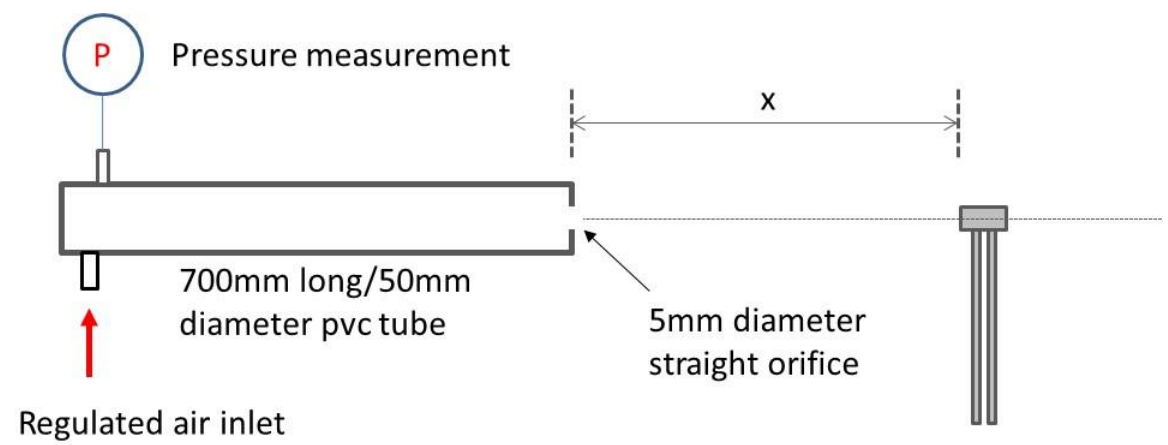

Figure 5: experimental device

A laser pointing from the closed end through the orifice traces out the exact location of the axis of the jet. Measurements were done along the axis of the jet at various distances from the orifice (between $10 \mathrm{~cm}$ and $100 \mathrm{~cm}$ ). The results presented below were obtained with air pressure in the chamber between 100 and $650 \mathrm{mbar}$ so that the exit velocity ranges from 120 to $300 \mathrm{~m} / \mathrm{s}$ with a Reynold number between 35000 and 90000.

Reference measurements were performed using a hot wire device (Figure 6) equipped with a 5 $\mu \mathrm{m}$ diameter, $1.25 \mathrm{~mm}$ long platinum-coated tungsten wire sensor. The signal conditioning electronics is a 54T42 MiniCTA single channel anemometer optimized for moderate-speed airflow (up to $100 \mathrm{~m} / \mathrm{s}$ ) operating at constant temperature. The upper cut off frequency is $10 \mathrm{kHz}$ at $50 \mathrm{~m} / \mathrm{s}$. Note however that this frequency is expected to depend on the average flow velocity (smaller with lower velocity) and on the intensity of the fluctuations (smaller with higher fluctuations).
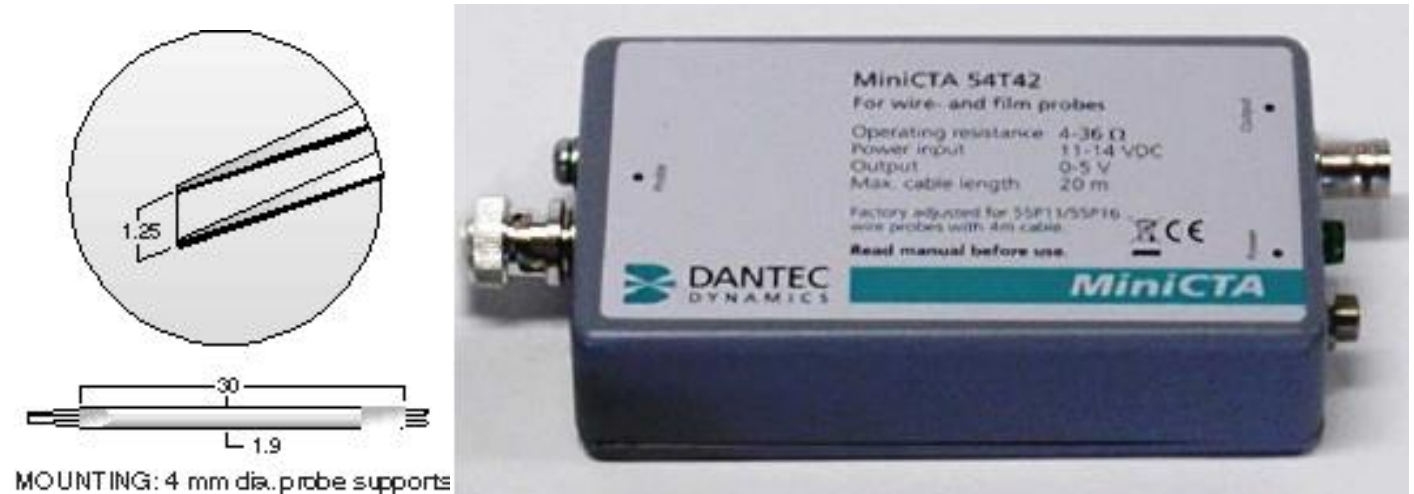

Figure 6: hot wire device used as a reference measuring device

Typical results are presented on figure 7 and 8 together with the theoretical correlations. Note that the auto correlation coefficient was obtained using the Taylor approximations (equation [9]) which might be a rough estimate in this "high turbulence case" but enough for comparison purposes. Thus, the space correlation function is deduced from the autocorrelation function in time ( $\mathrm{t}_{\text {meas }}$ is the duration of the signal and $\tau$ the advection time of the turbulence along a distance $\mathrm{x})$ :

$$
R(x=U \cdot \tau)=\frac{1}{t_{\text {meas }}} \cdot \int_{0}^{t_{\text {meas }}} \frac{u(t) \cdot u(t-\tau)}{u^{\prime 2}} \cdot d t \quad[9] \text { with } \mathrm{t}_{\text {meas }}>\tau
$$


The reader accustomed with signal analysis may be aware of the fact that the tools used to reduce the data and specially to obtain the spectrum might introduce significant difficulties. The reader is referred to appendix B for further details.
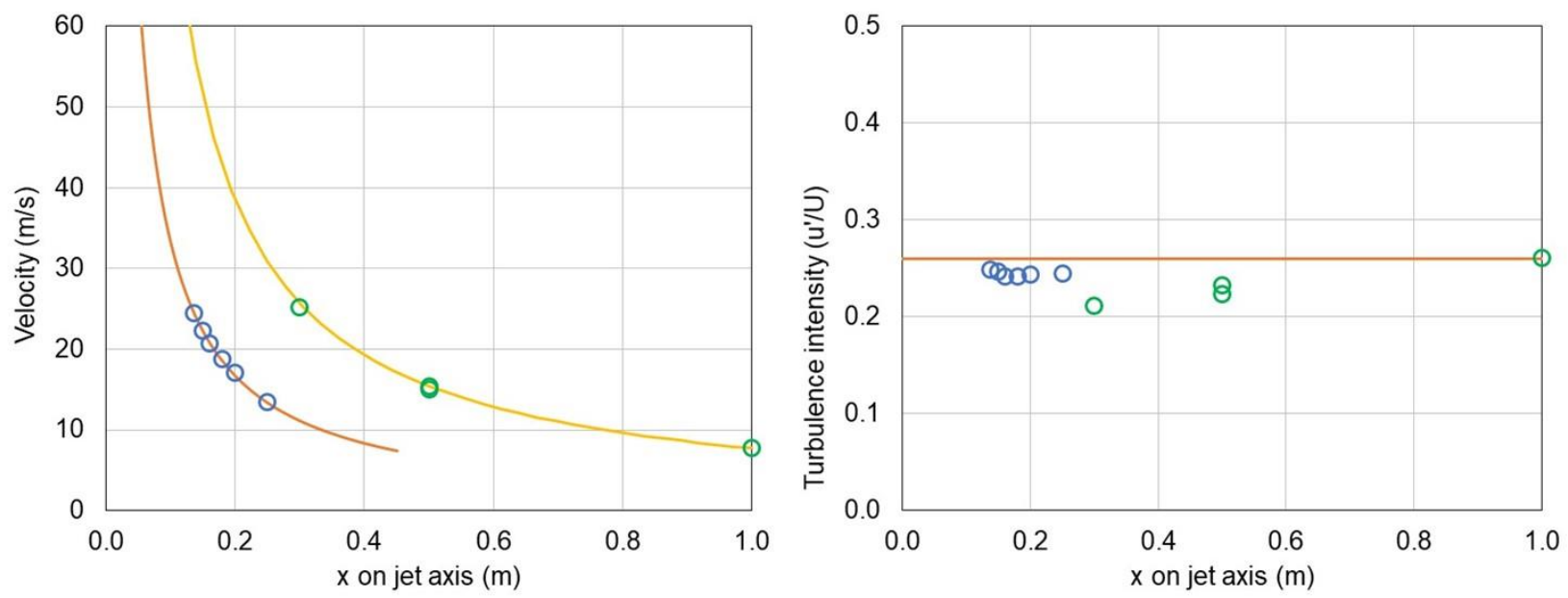

Figure 7: hot wire - average flow velocity and turbulence intensity as function of the release pressure (blue circle: 110 mbar release pressure, green circle: $585 \mathrm{mbar}$ ).
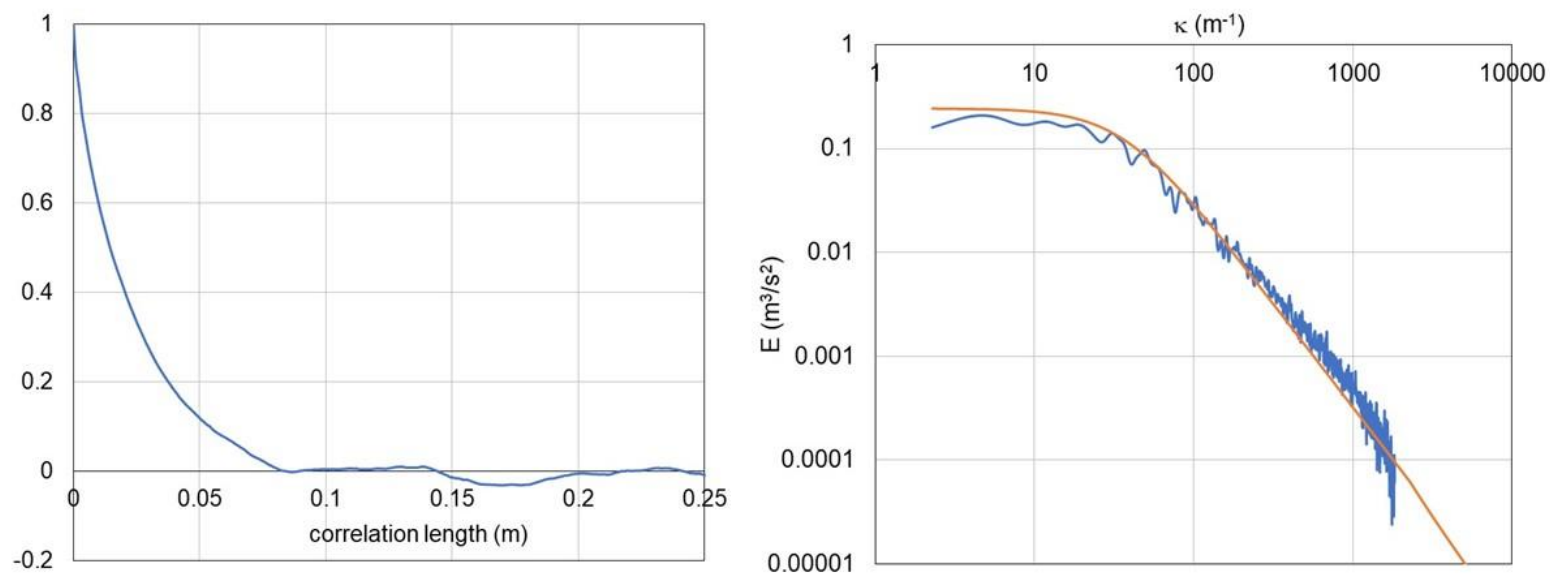

Figure 8: hot wire - longitudinal velocity correlations and associated turbulence spectrum at $50 \mathrm{~cm}$ from the orifice (585 mbar release pressure)

The integral length scale can be deduced from the correlation curves: $0.022 \mathrm{~m}$ at $50 \mathrm{~cm}$ from the orifice. This value is lower than expected $(0.025 \mathrm{~m}$ theoretically) using correlations [7]. Note that the turbulence intensity is somewhat too small too. Nevertheless, the turbulence spectrum is in good agreement with the theory (for hot wire used as a turbulence measurement standard see for example Wygnanski, 1969, and Wilson, 1964).

The spectrum is valid up to about $\kappa_{\mathrm{c}}=1200 \mathrm{~m}^{-1}$. At larger wavenumbers, the evolution of the spectrum is erratic suggesting that noise is predominant at large frequencies. The corresponding cut-off frequency, $f_{c}$, is $\kappa_{c}=2 . \pi . f_{c} / U$ where $U$ is the local mean flow velocity. $U$ is about $15 \mathrm{~m} / \mathrm{s}$ so that $\mathrm{f}_{\mathrm{c}}=3000 \mathrm{~Hz}$. It is believed that the cut-off frequency of the hot wire device was reached under the present experimental conditions (moderate average velocity and large fluctuations).

The influence of the cut-off scale on the measurement of $\mathrm{u}$ ' is discussed in appendix $\mathrm{C}$. In the present case, the theoretical wavenumber corresponding to the integral scale of the turbulence, 
$\kappa_{\text {int }}$, is $250 \mathrm{~m}^{-1}$ (for $\mathrm{L}_{\mathrm{int}}=0.025$ ). The ratio of $\kappa_{\mathrm{c}}$ with $\kappa_{\mathrm{int}}$ is then about 5 , so $\mathrm{u}$ ' might be slightly underestimated (about 1\%) which seems in line with the present results.

\section{Bidirectional Pitot probe performances}

The influence of the geometry of the bi-directional probes were investigated and especially that of the dimensions of the head (diameter/length) and that of the length of the pipes connecting the head to the sensor. The dimensions which were tested are presented on figure 9.

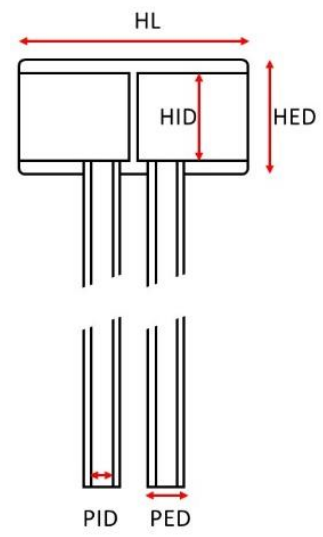

\begin{tabular}{|r|c|c|}
\cline { 2 - 3 } \multicolumn{1}{|c|}{} & \multicolumn{2}{|c|}{ Probe size $(\mathbf{m m})$} \\
\cline { 2 - 3 } & $\begin{array}{c}\text { Small } \\
\text { Head }\end{array}$ & $\begin{array}{c}\text { Medium } \\
\text { Head }\end{array}$ \\
\hline HL (head length) & 12 & 20 \\
\hline HED (head external diameter) & 8 & 12 \\
\hline HID (head internal diameter) & 5 & 9 \\
\hline PED (pipe external diameter) & 3 & 5 \\
\hline PID (pipe internal diameter) & 2 & 3 \\
\hline
\end{tabular}

Figure 9: Geometry and dimensions of the bidirectional probes submitted to the tests

The differential pressure transducers (Texence Sensor) which are implemented (figure 10) incorporate two separate micro-piezoelectric sensors (FirstSensor). The electronics is embedded. A standard filtered version is proposed with a reported cut-off frequency of $200 \mathrm{~Hz}$. But for the present application, non-filtered versions were used with a cut-off frequency expected to be larger than $500 \mathrm{~Hz}$. This point is addressed further later. Measurements were performed with a sampling rate of $5000 \mathrm{~Hz}$.

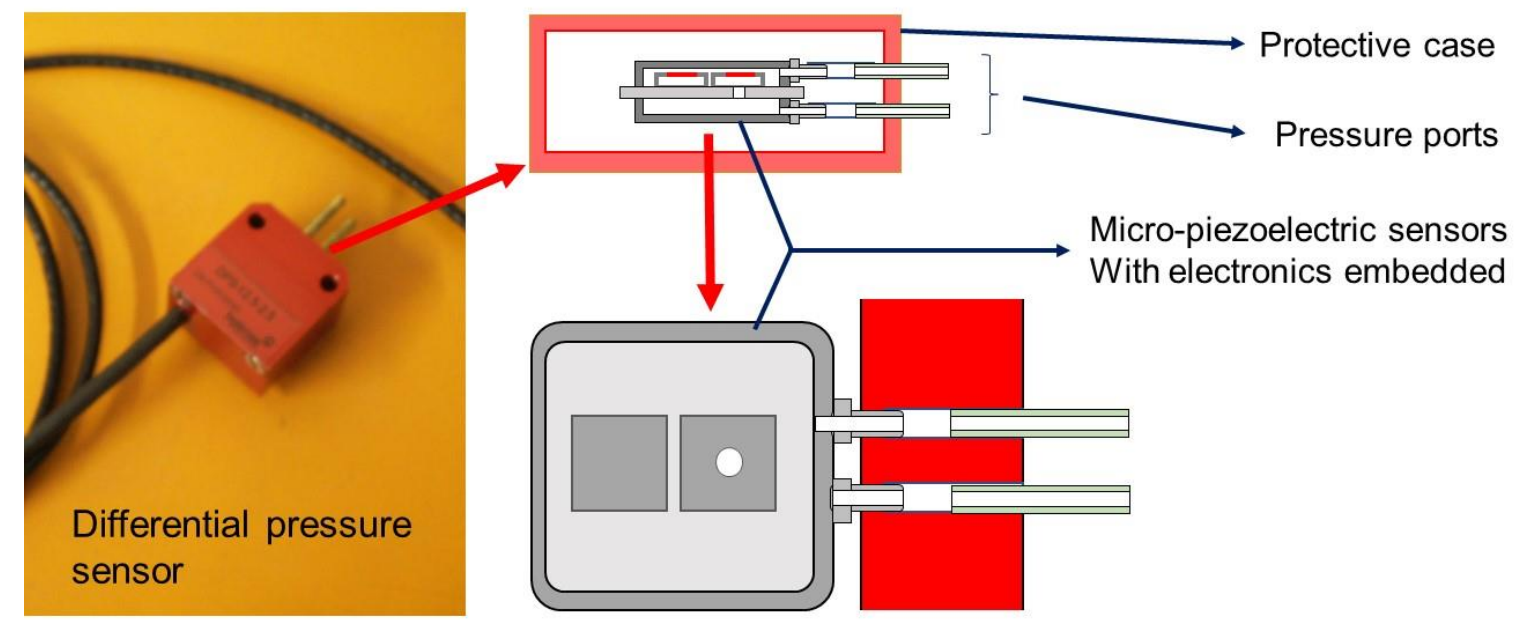

Figure 10: Differential pressure transducer used (left) and sketch of the piezoelectric microsensors

Examples of average velocities and turbulence intensities obtained in the reference jet are reported on figure 11 along with the theoretical correlations. The tests were performed under the same release conditions than for the hot wire but with different probes having various pipe 
lengths and head sizes. It may be observed that even if the mean velocity is in line with correlations, the intensity of turbulence may deviate significantly from theory. There is thus a need to clarify the response of the probes.
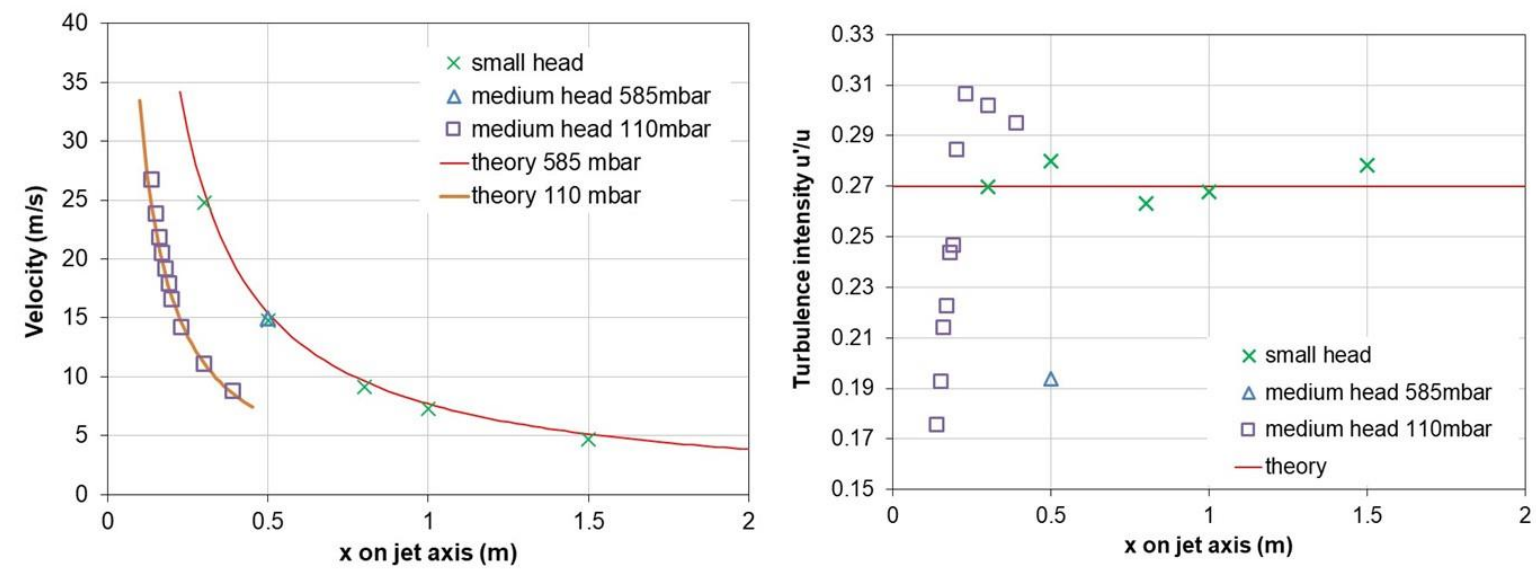

Figure 11: average flow velocity and turbulence intensity as function of the release pressure and probe head geometry

Actually, the various autocorrelation spectra (obtained at $50 \mathrm{~cm}$ from the orifice on the axis of the jet) presented on figure 12 (same conditions than for figure 8-hot wire) show that the effect of the pipe length connected to a medium head is significant.
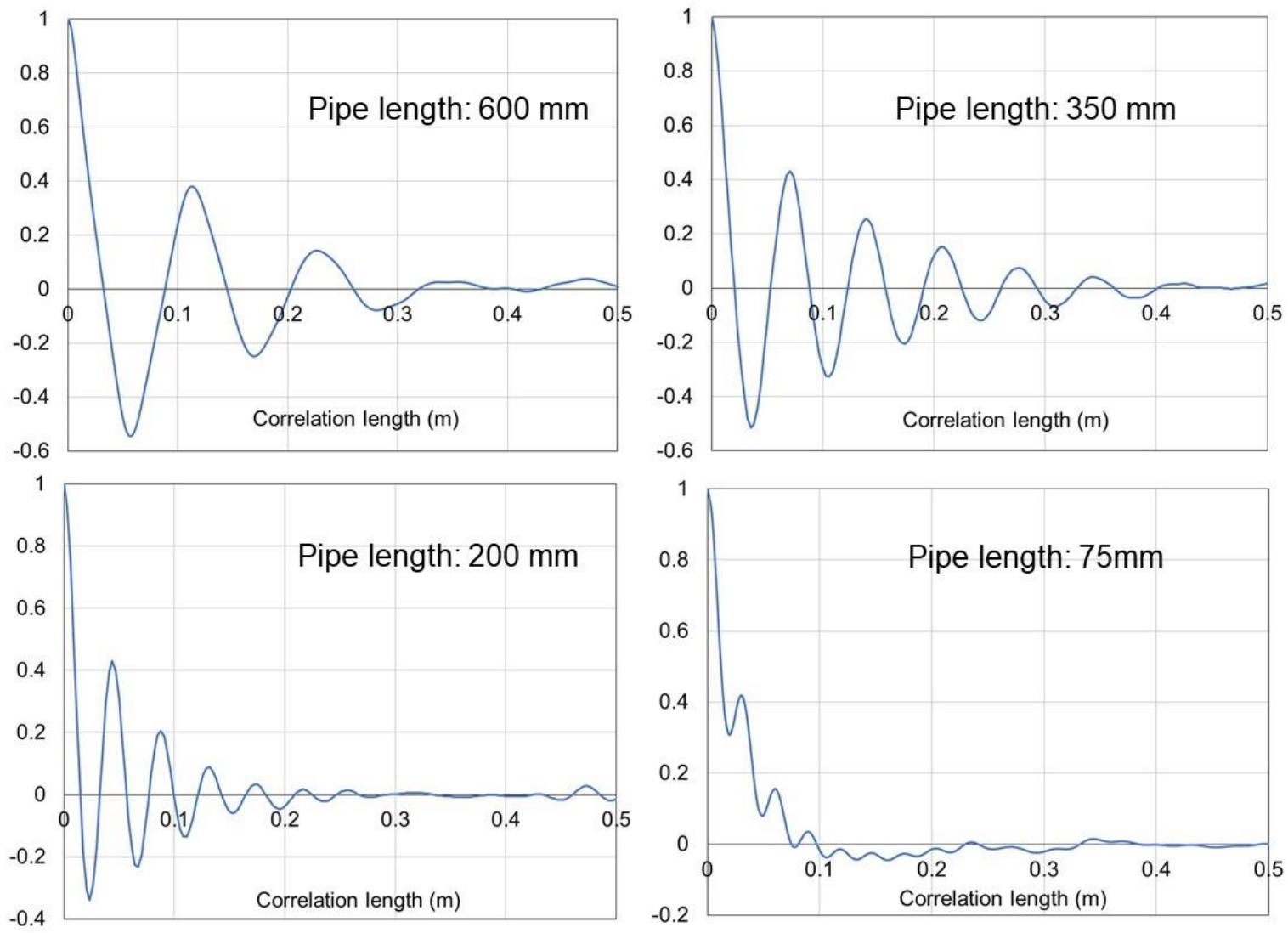

Figure 12: longitudinal velocity correlation obtained at $50 \mathrm{~cm}$ from the orifice (585 mbar) for various pipe length (medium head) 
As compared to the reference autocorrelation spectra obtained with the hot wire (figure 8), a sort of sinewave signal is superimposed over the expected exponential decay. This additional signal does not seem to be related to the turbulence, but its sinusoidal behavior suggests an acoustic/aeraulic phenomenon. The longer the pipes of the head, the larger this effect.

\section{Acoustics and aeraulics}

A strong effect of this on the wavenumber spectrum is manifested with a clear bump appearing at medium wavenumbers (figure 13).

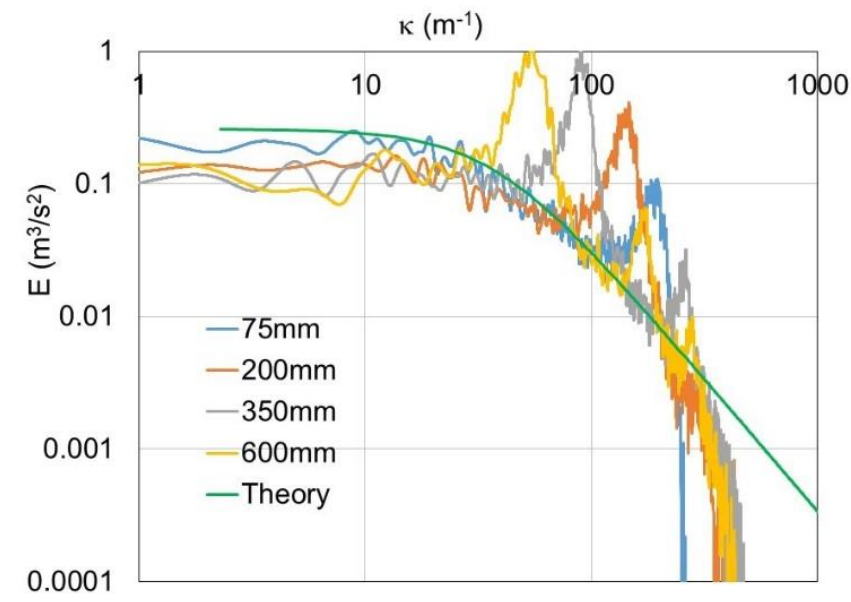

\begin{tabular}{|l|l|l|l|l|}
\hline $\begin{array}{l}\text { Medium } \\
\text { Head }\end{array}$ & $\begin{array}{l}\text { Pipe Length } \\
(\mathrm{mm})\end{array}$ & $\begin{array}{l}\mathrm{U} \\
(\mathrm{m} / \mathrm{s})\end{array}$ & $\begin{array}{l}\mathrm{u}^{\prime} \\
(\mathrm{m} / \mathrm{s})\end{array}$ & $\mathrm{u}^{\prime} / \mathrm{U}$ \\
\hline 75 & 14,7 & 3,1 & 0,21 \\
\hline 200 & 14,6 & 4,7 & 0,32 \\
\hline 350 & 14,7 & 5,4 & 0,37 \\
\hline 600 & 14,5 & 5,3 & 0,38 \\
\hline
\end{tabular}

Figure 13: turbulence spectra corresponding to the longitudinal velocity correlation of figure 12. $U, u$ ' and turbulent intensity in the table

For the shorter pipe length $(75 \mathrm{~mm})$, only a weak interference is seen, in contrast with the results obtained with the $600 \mathrm{~mm}$ pipe length for which the resonance frequency "bump" overlaps to a large extent the autocorrelation trace. Most often, this bump is followed by a significant drop of the spectrum.

The effect of the pipe length is very similar with a small head (figure 14). But when comparing figure 13 and 14, it seems that the head size has also some impact on the resonance frequency (Compare for example the medium head $-200 \mathrm{~mm}$ and the small head $-180 \mathrm{~mm}$ ).

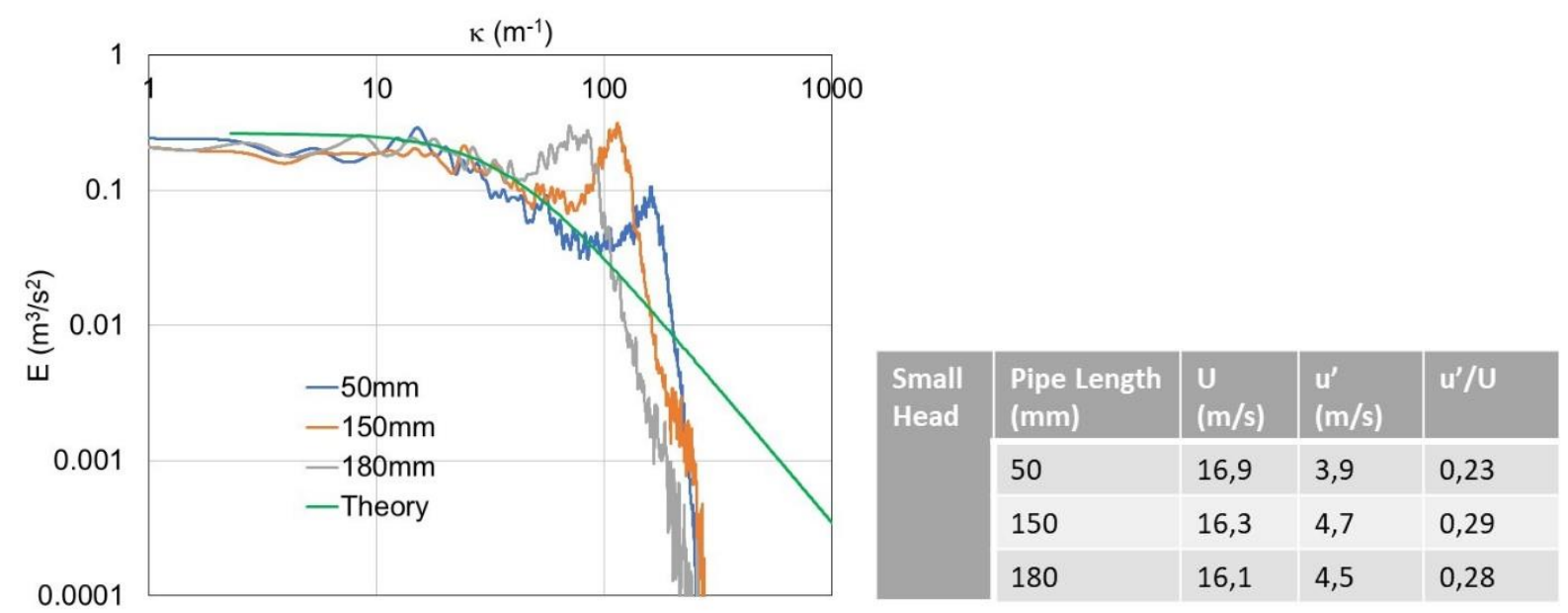

Figure 14: turbulence spectra obtained with a small head for various pipe lengths. $U, u$ ' and turbulent intensity in the table. 
This resonance influences the turbulence energy distribution, with a significant bump in the energy containing eddy scales. The total energy might look greater than contained in the theoretical spectrum, explaining why turbulence intensity is sometimes larger than expected. In other situations, when the "bump" is less pronounced, the drop of the spectrum (usually following the bump) may cause a lower than expected turbulence level.

The nature and the source of the interference needs to be clarified. There is clearly a link with the geometry. It could be a pure aeraulic phenomenon (aero elastic/spring-mass, aero acoustic) or an acoustic one (resonance in the piping). Our tests (figure 15 with spectra given in $\mathrm{Hz}$ and not in wavelengths) show that the speed of the flow does not seem to affect the location and intensity of the "bump" suggesting the aero acoustic option is improbable. In appendix D, the assumption of a mechanical vibration of the head+pipes system due to the flow is investigated. For the situation of figure 15, typical resonance frequencies of $500 \mathrm{~Hz}$ are found which are comparable with those corresponding with the "bumps" but, due to the stiffness of the device, the displacements produced by the flow $(10-20 \mathrm{~m} / \mathrm{s})$ are very small and correspond to undetectable velocities $(0.001 \mathrm{~m} / \mathrm{s})$. No bump can be produced. Following, the interference phenomena is certainly due to the acoustics of the head+pipe+sensor arrangement. An acoustic model of the device is presented in appendix E. Each branch is modelled as a series of interconnected tube assigned to impedances. The model, although crude, reproduces rather faithfully the measured resonance frequencies (Figure 16).

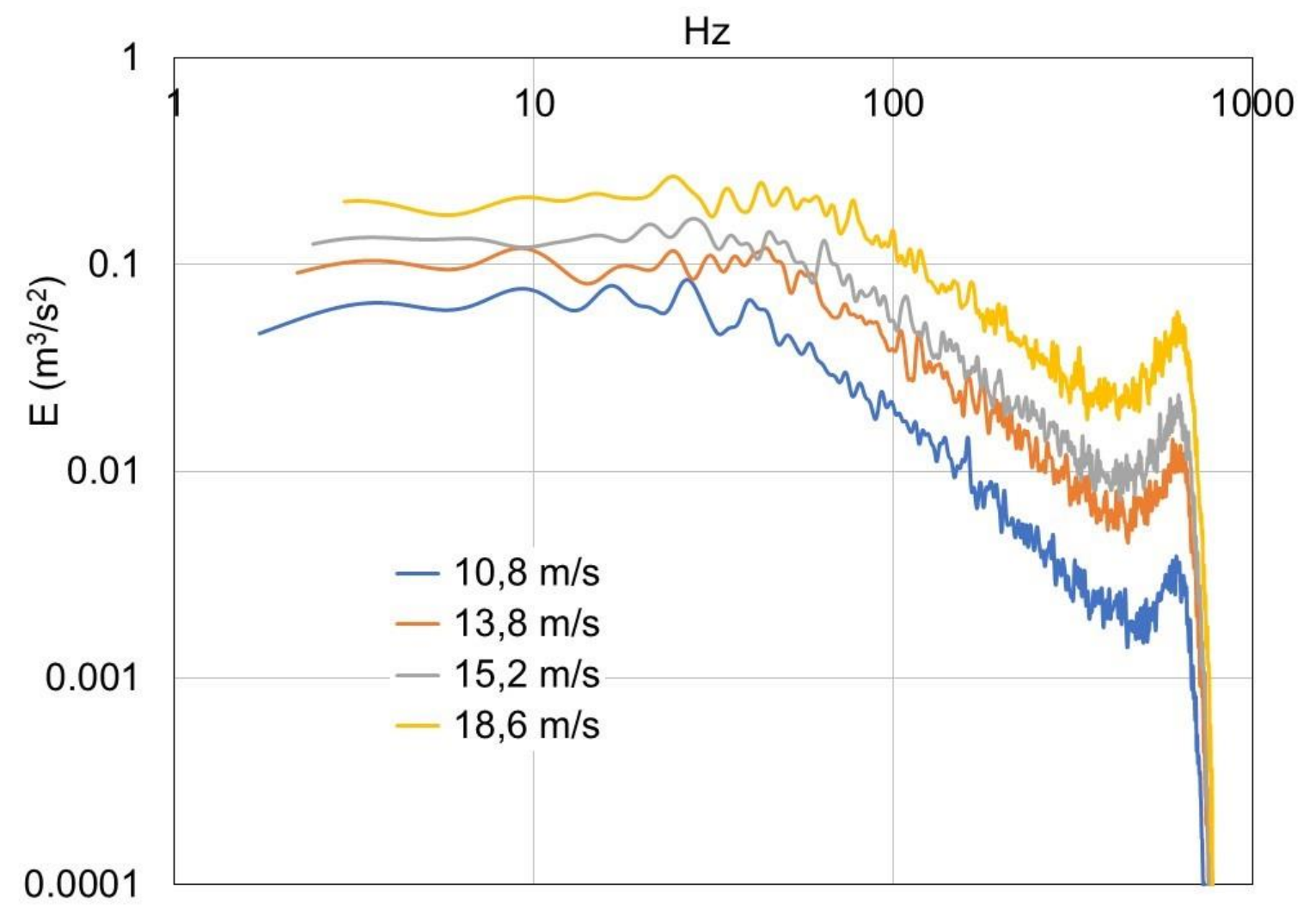

Figure 15: Influence of the flow velocity on the turbulence spectra at 50cm from the orifice (velocity changes as function of the stagnation pressure). $\mathrm{X}$-axis expressed in $\mathrm{Hz}$ (medium head- 75 mm pipe length) 
This proves that the "bumps" on the turbulence spectra are due to the internal acoustics of the probe. Further analysis is proposed in appendix E about the "best" design. It shows up that "small" heads are not the most suitable. Medium head would be preferable provided a short enough pipe is used (less than $100 \mathrm{~mm}$ long).

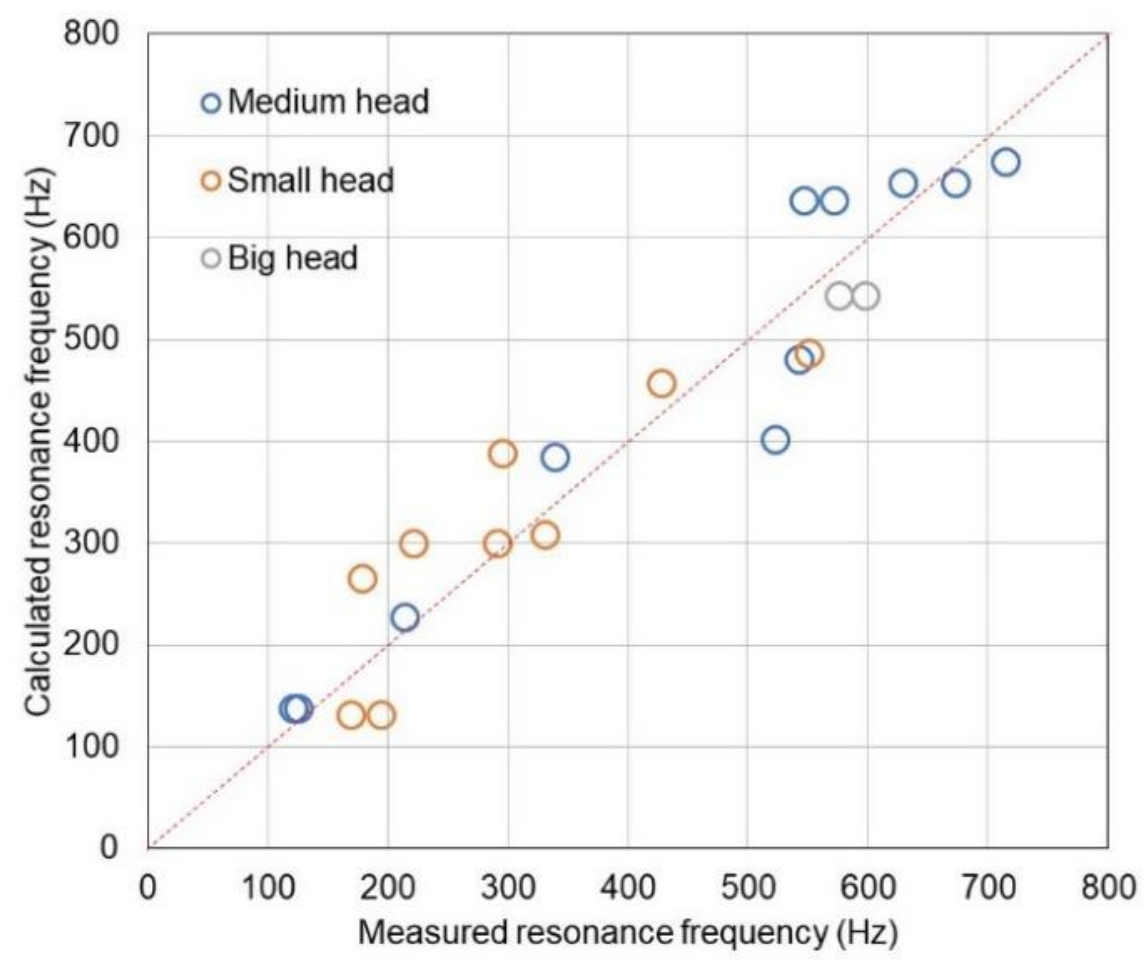

Figure 16: Calculated versus measured resonance frequencies using the acoustic model presented in appendix E (the "big head" cases are introduced in appendix E)

An example is given on figure 17 showing the performance of a "best practical" design of such a probe. The turbulence spectrum is now resembling that obtained with the hot wire. A very small bump remains followed by a sharp drop at about $300 \mathrm{~m}^{-1}$. The corresponding frequency is $700 \mathrm{~Hz}$. Big heads could be used but they are more intrusive and more difficult to clamp. 


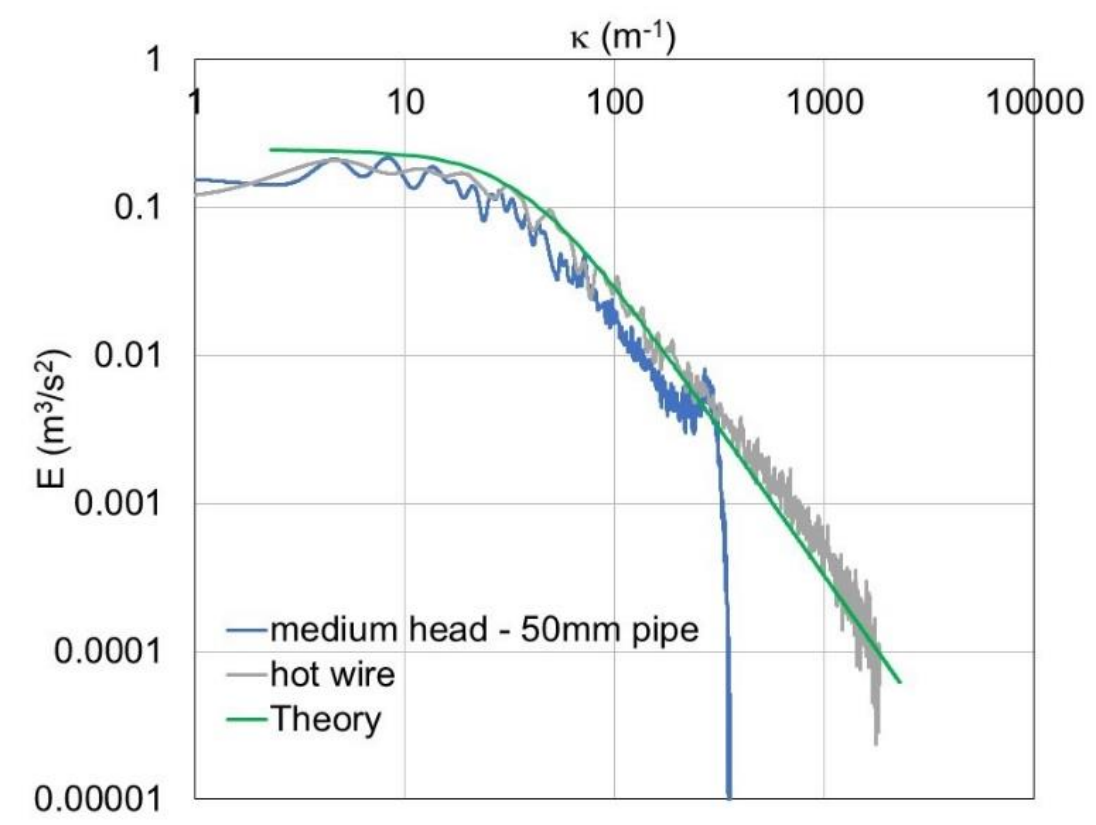

Figure 17: Comparison of turbulence spectra between the hot wire and a "best practical" design of the bidirectional probes (medium head-50 mm pipe). Stagnation pressure of 580 mbar. Measurement at $50 \mathrm{~cm}$ from the orifice on the axis.

Although this cut off wavenumber may look large enough to capture most of the spectrum, it should be noted that, in the present jet configuration, the wavenumber corresponding to the integral scale of the turbulence is $280 \mathrm{~m}^{-1}$, not so far from the cutoff wavenumber. Using the results of appendix $\mathrm{C}$ reveals that $\mathrm{u}^{\prime} / \mathrm{U}$ might be underestimated by almost $5 \%\left(\wedge_{\text {cut-off }} / \Lambda_{\text {int }} \approx 1\right)$. But remember also that, by design, the measured value of $u^{\prime}$ is underestimated also by typically $10 \%$ (section 3). It can therefore be expected that the measured value of $\mathrm{u}^{\prime} / \mathrm{U}$ is underestimated by $15-20 \%$ which is indeed the case ( 0.21 as compared to 0.26$)$.

\section{Practical implications}

So, this "best practical" design probe could perform well only when the integral scale is large enough because of the resonance phenomenon described above but also because the intrusive nature of the head may disturb the eddies.

In order to illustrate this point, a bidirectional probe (small head-600 $\mathrm{mm}$ pipe) was moved along the axis of the jet and the turbulence intensity ( $\mathrm{u}^{\prime} / \mathrm{U}$ ) was measured (figure 18). At $20 \mathrm{~cm}$ from the orifice, the turbulence intensity drops suddenly while it was shown browsing with the hot wire that it should be constant ${ }^{2}$. This can only be due to the various limitative phenomena described above. Note in the present situation, at $20 \mathrm{~cm}$ from the orifice, $\mathrm{L}_{\text {int }}$ is on the order of $1 \mathrm{~cm}$, close to the size of the head.

\footnotetext{
${ }^{2}$ It can be shown that the turbulence intensity should be constant from about $20 \mathrm{~mm}$ downstream (Ball 2012)
} 


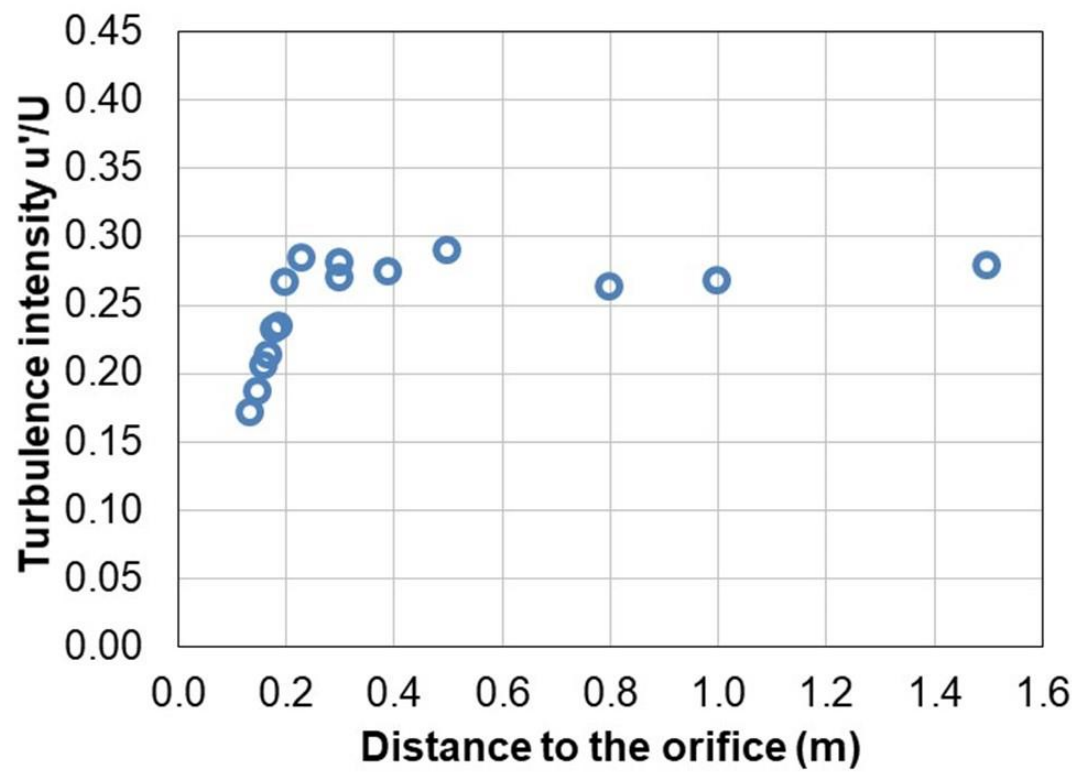

Figure 18: Turbulence intensity according to the position of the probe along the $x$-axis (100 mbar in the stagnation reservoir).

Apart from the very peculiar situation in laboratory testing where the size of the probe might be comparable to that of the largest eddies, the limitation will probably be brought in field experiments by the frequency cut-off. A clear drop of the performance is expected to be reached (appendix C) when the cut-off length scale $\left(\mathrm{L}_{\text {cut-off }}=\mathrm{U} / \mathrm{f}_{\mathrm{c}}\right)$ of the probe is such that $\mathrm{L}_{\text {int }} / \mathrm{L}_{\text {cut-off }}<0.5$ or equivalently when $\mathrm{f}_{\mathrm{c}}<0.5 \mathrm{U} / \mathrm{L}_{\text {int }}$.

The highest performance and robustness for the probe is that of a medium head with 50-100 $\mathrm{mm}$ long connecting pipes. The head is not too intrusive, the pipes are short but still long enough to enable a clamping on a support and the pressure detector can be protected from the flame. An example is shown on figure 19.

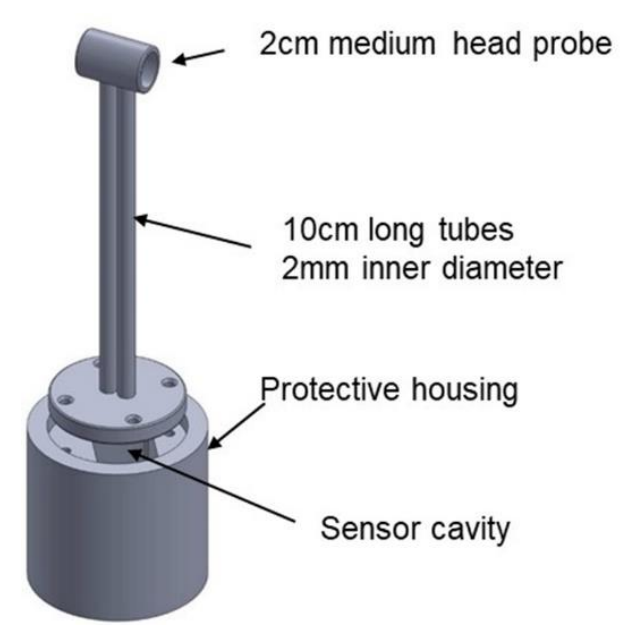

Figure 19: drawing of a probe designed for large scale experiment in harsh environment

The cut-off frequency is the main limitation and the latter is due to the acoustics of the probe. The pressure sensor offers a higher cut-off frequency. It was attempted to simplify drastically 
the acoustics by integrating the pressure transducer in the probe head (pipes have been removed, figure 20).

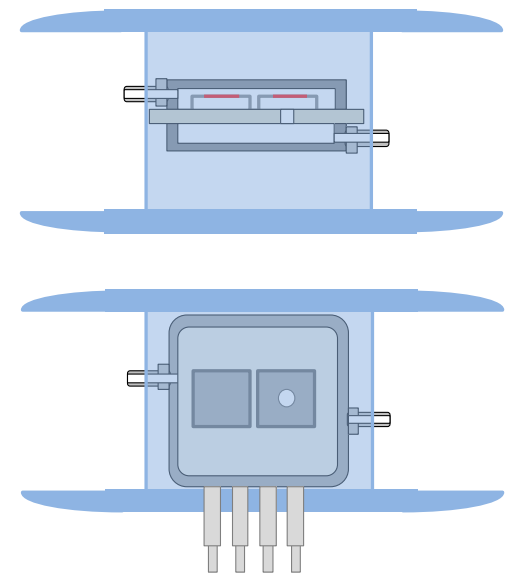

Figure 20: schematic view of a prototype integrating the pressure transducer directly inside the bidirectional head

The space correlation curves are compared with that obtained with a medium head/short pipes bidirectional probe, on one end, and with the hot wire on the other end (figure 21). The curve obtained with this new tentative design is nearly superposed with that of the hot wire.

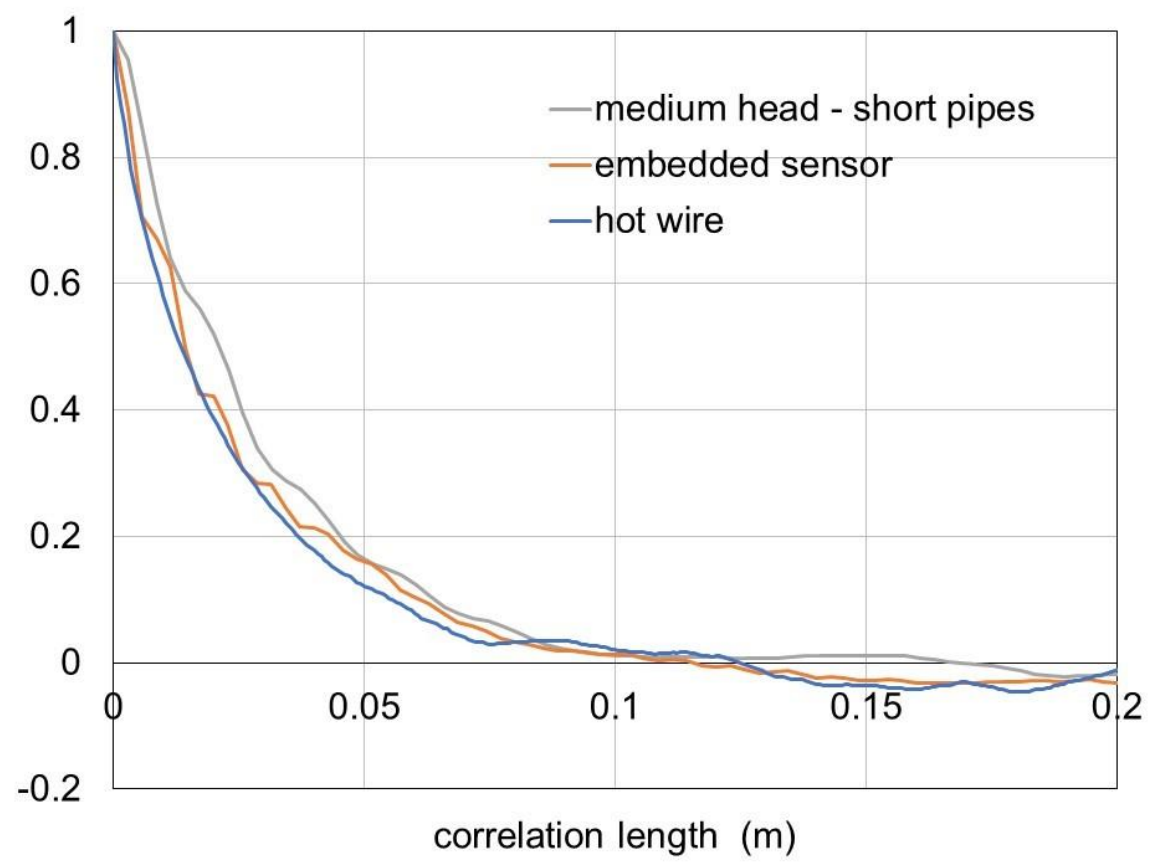

Figure 21: Comparison of space correlation curve: hot wire, embedded sensor (prototype) and bidirectional probe (50cm from the orifice, $585 \mathrm{mbar}$ ).

The turbulence spectra are shown on figure 22. A residual resonance (and cut-off) appears for the embedded sensor at a wavelength of $600 \mathrm{~m}^{-1}$, so nearly twice higher than for the "best design" bidirectional probe. The corresponding cut-off frequency is $1300 \mathrm{~Hz}$.

Considering the estimated volume surrounding the piezoelectric micro sensor $\left(75 \mathrm{~mm}^{3}\right)$ and the size of the small channel (diameter: $0.5 \mathrm{~mm}$, length: $5 \mathrm{~mm}$ ) connecting the sensor cavity to the 
outlet tubes, an Helmholtz frequency of about $1250 \mathrm{~Hz}$ is estimated, which could correspond to the resonance observed at $1300 \mathrm{~Hz}$.

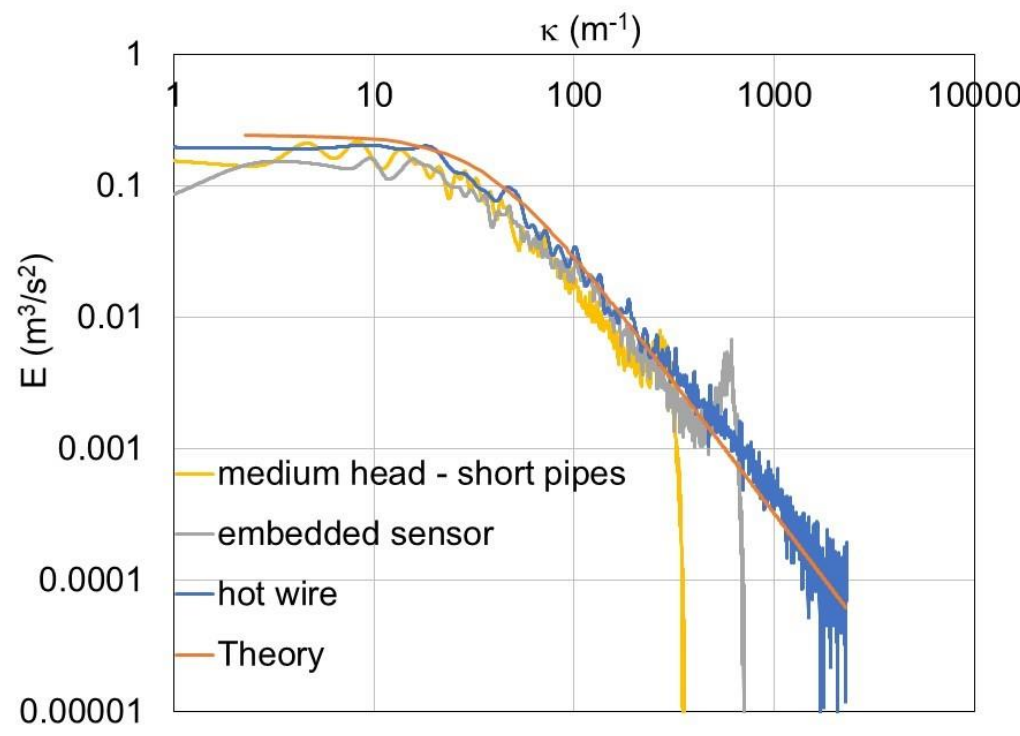

Figure 22: Comparison of turbulence spectra: hot wire, embedded sensor (prototype) and bidirectional probe (data from figure 21).

\section{Conclusions and perspectives}

Within the frame of industrial leakages with large flowrate, large turbulence scale, biphasic flows, important density gradients, the bidirectional probe concept offers significant robustness and might be one of the only mean to measure turbulence.

In this paper, the theory behind this concept is explained, the performances and the limitations are discussed by comparison with reference hot wire measurements. It was shown that the volume of the head of the sensor may interact with the local turbulent eddies and that some acoustic resonance may superpose on the turbulence signals affecting the turbulence spectrum.

A good compromise was obtained using a $2 \mathrm{~cm}$ long, $1 \mathrm{~cm}$ diameter head connected to the pressure sensor via 50-100 mm long pipes. The cut-off in frequency is about $700 \mathrm{~Hz}$ without significant resonance. The head of the probe is expected to interact significantly on the turbulent eddies only when the integral scale of the turbulence is similar to the diameter of the head. This is not a limitation for field experiments for which this kind of probe is principally designed for.

A robust design is shown on figure 20 above and was used extensively in our test site in these last years (Sail et al, 2014). It is believed that good estimates of the turbulence intensity can be achieved provided the mean flow is such that $U / L_{i n t}<2 . f_{c}$ where $f_{c}$ is the cut-off frequency of the probe $(700 \mathrm{~Hz})$

Certainly, improvements are possible but would require a profound redesign of the head of the probe and of the pressure sensor. But in many practical field experiments, the present design is already a very useful tool.

\section{References}


BALL C. G., FELLOUAH H., POLLARD A. (2012), “The flow field in turbulent round free jet "- Progress in Aerospace Sciences vol 50, pp 1-26

BECKER H. A., BROWN A. P. G. (1974), "Response of pitot probes in turbulent streams" Journal of Fluid Mechanic (1974), vol 62, part1, pp 85-114

BIRCH A.D, BROWN D.R., DODSON M.G., SWAFIELD F. (1983), "The structure and concentration decay of high-pressure jets of natural gas" - Comb. Sci. and Techn., vol. 36, pp. 249-261

CARAZZO G., KAMINSKI E., TAIT S. (2006), "The route to self-similarity in turbulent jets and plumes" - J.Fluid Mech.vol. 547, pp 137-148

CORTESE, PERLEE H. (1992), "Robust bi-directional turbulence probe" - Rev. Sci. Instrum. 63 (7), july 1992 pp3729-3732

DJERIDANE T. (1994), «Contribution à l'étude expérimentale des jets turbulents axisymétriques à densité variable » - Thèse de doctorat, Université de Marseille

EU-Project DESC (Contract N G1RD-CT-2001-00664)

HINZE J.O. (1975), “Turbulence” - Mc GrawHill, New-York

JEZDINSKY V. (1966), "Measurement of turbulence by pressure probes" - AIAA Journal, vol. 4

KENT L. A., SCHNEIDER M. E., (1987), "the design and application of bi-directional velocity probes for measurements in large pool fires" - Instrum. Soc. Of America vol 26, n4 (1987) pp25-32

LIU C. Y., WONG Y. W., CHAN W. K., GAN T. C. (1990), "Note on the robust bi-directional low velocity probe" - Experiments in fluids 9 (1990) pp354-356

MOODIE K. (1990), "Jets discharging to atmosphere" - Loss Prev. Process Ind., Vol. 3

Mc CAFFREY et al. (1976), "A robust bidirectional low-velocity probe for flame and fire application" - Comb. and Flame, vol. 26

SAIL J., BLANCHETIÈRE V., GÉNIAUT B., OSMAN K., DAUBECH J., JAMOIS D., HEBRARD J. (2014), "Review of knowledge and recent works on the influence of initial turbulence in methane explosion" - Tenth International Symposium on Hazards, Prevention, and Mitigation of Industrial Explosions - Bergen, Norway, 10-14 June 2014

SETTE B. J. G. (2005), "Critical considerations on the use of a bi-directional probe in heat release measurements » - Fire Mater. 2005; vol 29 ; pp 335-349

SETTE B. J. G. (2006) "Development of velocity pressure probe" - Fire and Materials 2006; 30; pp397-414

SHAUGHNESSY E. J., MORTON J. B. (1977), «Laser light-scattering measurements of particle concentration in a turbulent jet »- Journal of Fluid Mechanics , Vol 80, Issue 1, 4 April 1977 , pp. $129-148$

TALBOT B.(2009) « Mélange et dynamique de la turbulence en écoulements libres à viscosité variable » Thèse INSA Rouen

TAMANINI F. (1990) «Turbulence effect on dust explosion venting » Plant/Operation progress, Vol 9

TAMANINI F. et al. (1998), «Turbulent unvented gas explosions under dynamic mixture injection conditions » - 23rd Symp (Int.) on Com., pp. 851-858

TENNEKES H., L. LUMLEY J. L. - A First Course in Turbulence - MIT Press 1972

WILSON R. A. M., DANKWERTS P. V. (1964), "Studies in turbulent mixing-II: A hot-air jet »- Chemical Engineering Science, Vol 19, Issue 11, November 1964, pp 885-895 
WYGNANSKI I., FIEDLER H. (1969), Some measurements in the self-preserving jet » - J. Fluid Mech. (1969), vol 38, part 3, pp 577-612

YUN B. J., EUH D. J., KANG K. H., SONG C. H., BAEK W. P. (2005), "Measurement of the single- and two-phase flow using a newly developed average bi-directional flow tube" Nuclear Engineering and technology, vol37, n6 December 2005 
Appendix A: Aerodynamics of bidirectional probes

A large-scale low velocity wind tunnel is operated to evaluate and calibrate commercial devices used to measure gas flow velocities (figure A1). The flow velocity can be varied between 0.1 and $20 \mathrm{~m} / \mathrm{s}$ which correspond to a lot of industrial situations like tunnel ventilation, industrial building air renewal, exhaust hood,...

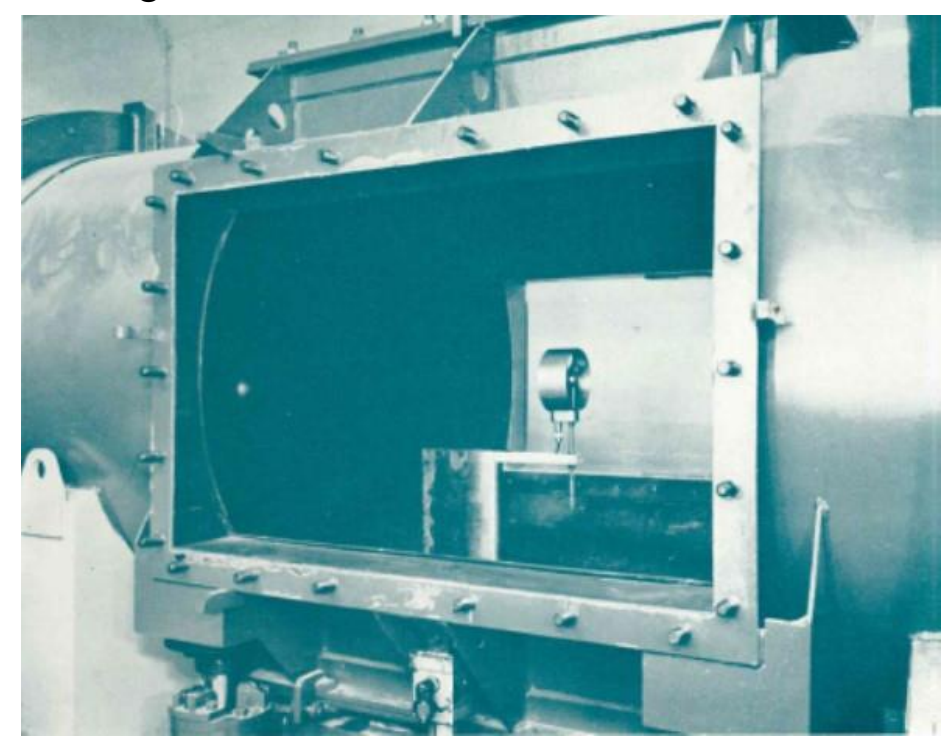

Figure A1: The low velocity wind tunnel of INERIS. Detailed view of the test chamber

This facility was used to evaluate the ability of the bidirectional probe to measure velocity in various conditions. The effect of the alignment of the probe in the flow was examined. The influence of the geometry of the head probe (size, proportion) was also investigated.

The compliance of this wind tunnel to ventilation standards is obtained using a referenced pitot tube. The measured physical value is then a differential pressure (dynamic pressure of the flow $\left.\mathrm{P}_{\text {dyncal }}\right)$ with an uncertainty of $+/-0.2 \mathrm{~Pa}$. It can be compared to the value of the dynamic pressure measured by the probe $\left(\mathrm{P}_{\text {dynexp }}\right)$.

An example is presented on figure A2. A rather good estimate of the dynamic pressure is provided by the bidirectional probe above $0.5 \mathrm{~Pa}$. This threshold depends strongly on the pressure sensor. The latter is often about $0.2 \%$ of the full range $(150 \mathrm{~Pa}$ full range in the present case).

The relation $P_{\text {dyncal }}=K \cdot P_{\text {dynexp }}$ obtained with $K=1.5$ is typical (see for example LIU, 1990, and YUN, 2005). K may vary a little according to the geometry of the probe in a similar way to the drag coefficient $\mathrm{Cx}$. The scattering of $\mathrm{K}$ reflects the uncertainty of the measurement of the dynamic pressure. In the present situation, this relative uncertainty is about $+/-8 \%$.

The dynamic pressure is directly linked to the velocity through: $\Delta P=\frac{1}{2} \cdot \rho \cdot U^{2}$, and the relative uncertainty on the velocity is then about $+/-5 \%$. Similar to figure A2, the comparison of the velocities can be obtained as shown on figure A3. 


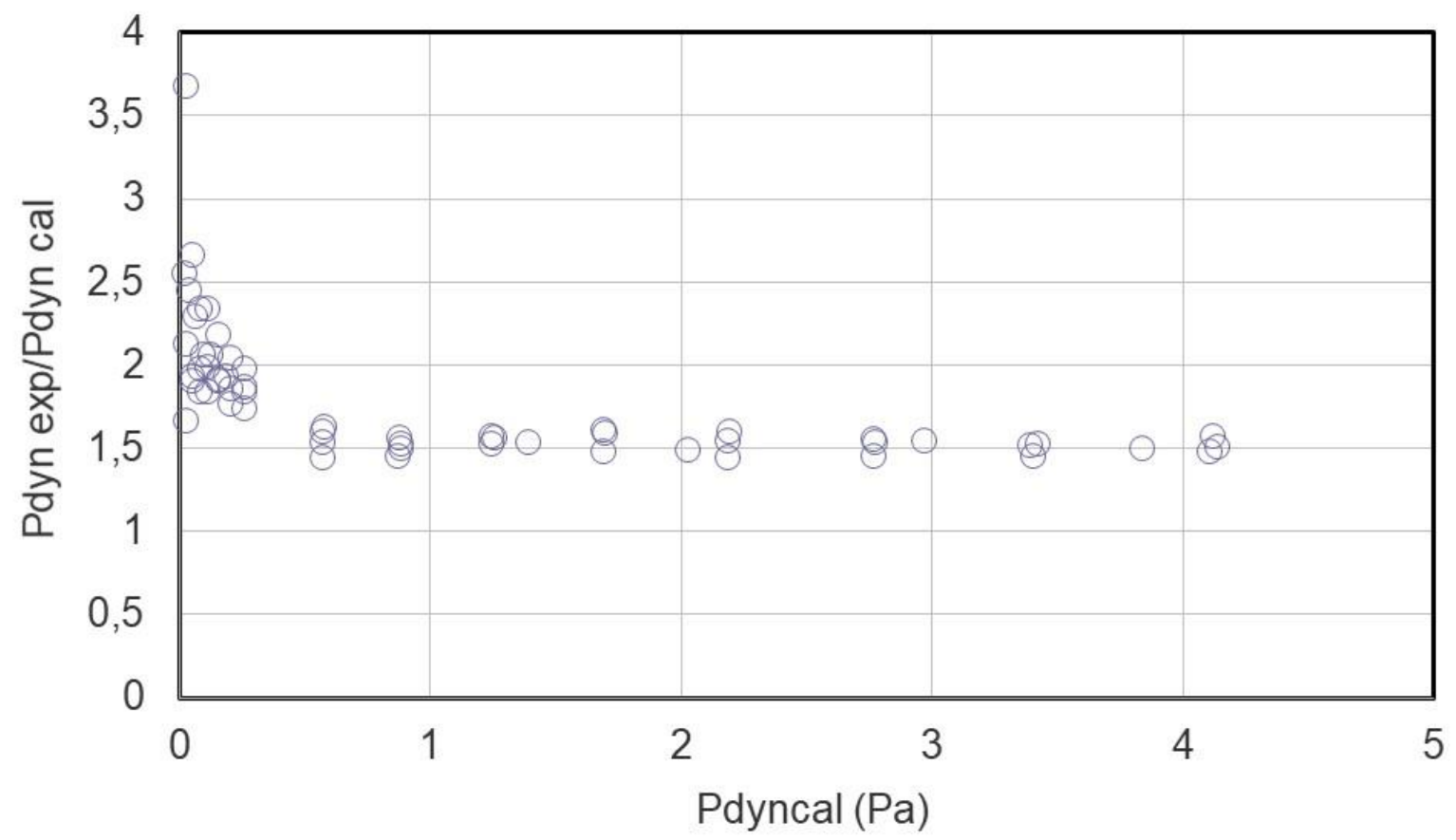

Figure A2: Dynamic pressure calibration curve obtained with a bidirectional probe in the wind tunnel

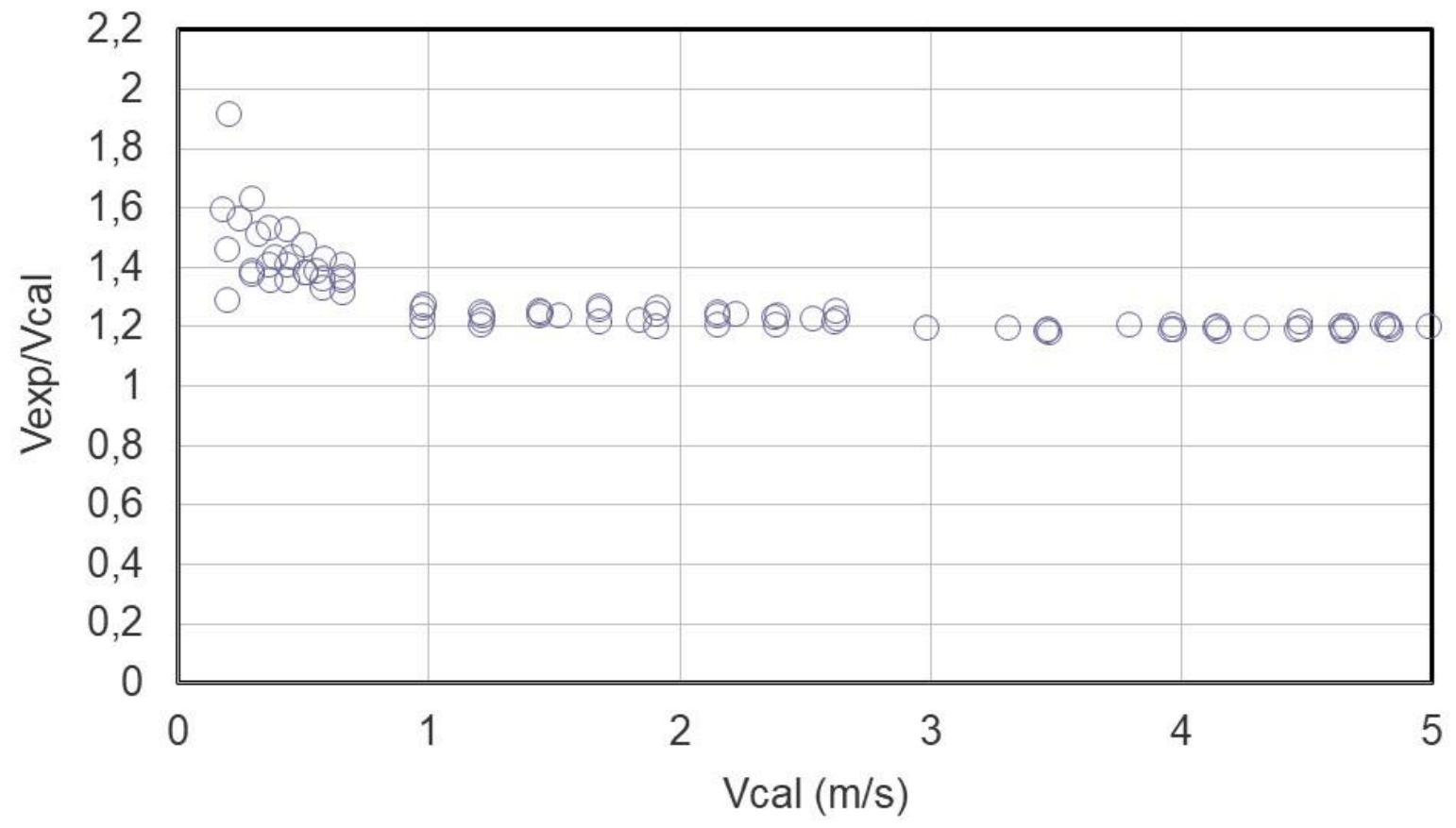

Figure A3: Velocity calibration curve obtained with a bidirectional probe in the wind tunnel (from the data of figure A2)

The relation $V_{\text {cal }}=C \cdot V_{\text {exp }}$ obtained with $C=1.2$ is typical too. This coefficient has been used systematically to convert the dynamic pressure raw data in velocities all along the present work.

Another issue frequently documented (SETTE, 2005) is the influence of the deviation of the head away the direction of the flow. A typical result is presented on figure A4. Taking account of the uncertainty, it appears that bidirectional probes are insensitive to the angle of deviation up to $40 / 50^{\circ}$. 


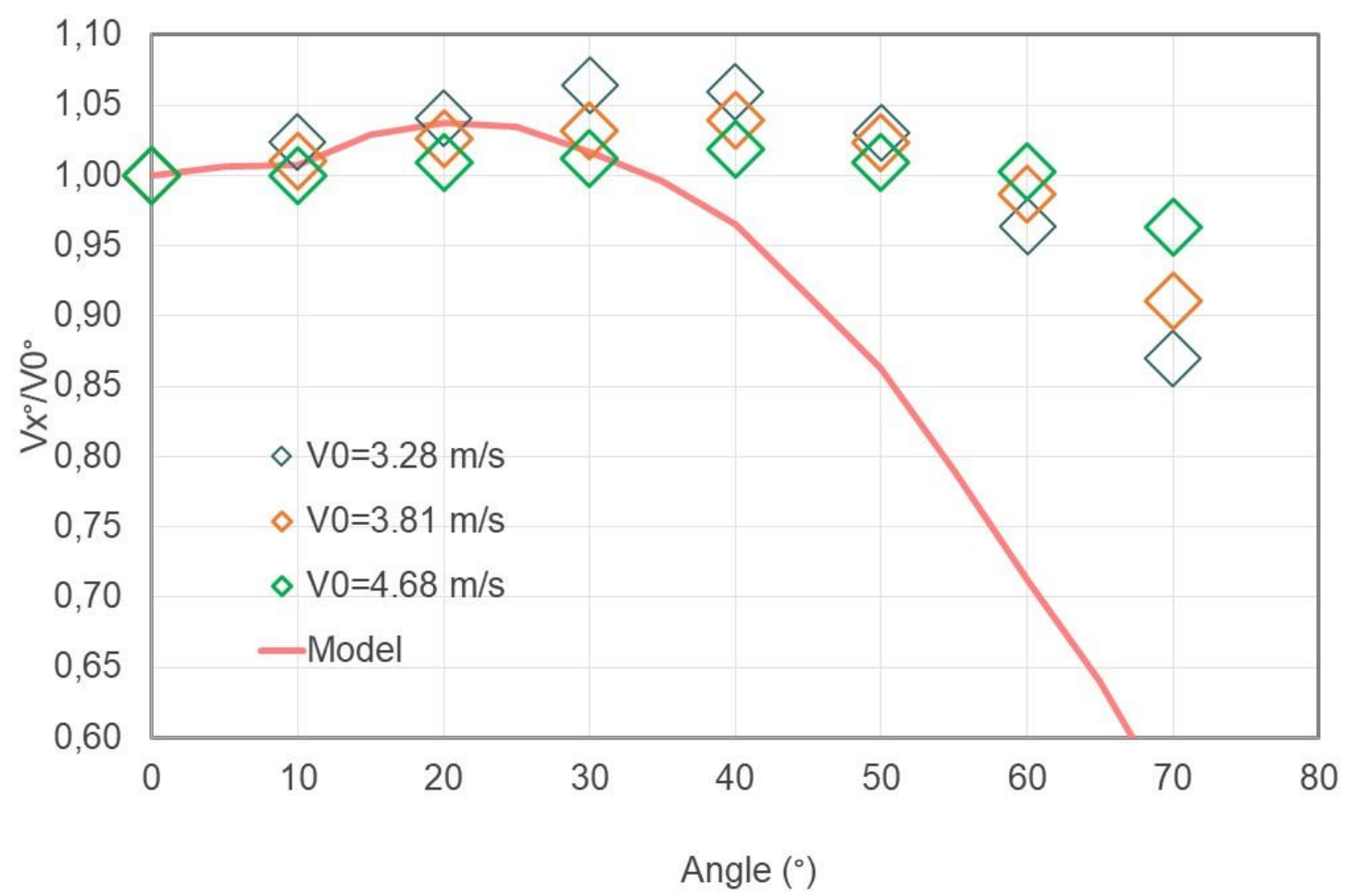

Figure A4: velocity measured with a medium head according to the angle toward the direction of the flow. Diamond size is proportional to the uncertainty along y axis.

On figure A4 is superimposed a curve obtained using a numerical model of the probe in the flow. COMSOL tool was used for this. The equations solved are that of the Reynolds-averaged Navier-Stokes (RANS) model for the conservation of momentum and the continuity equation for the conservation of mass. Turbulence effects are modelled using the standard two-equation $\mathrm{k}-\varepsilon$ model with realizability constraints for a weakly compressible single-phase flow at relatively high Reynolds numbers (figure A5). 2D axisymmetric flow is assumed with 38000 cells (mesh convergence was checked)

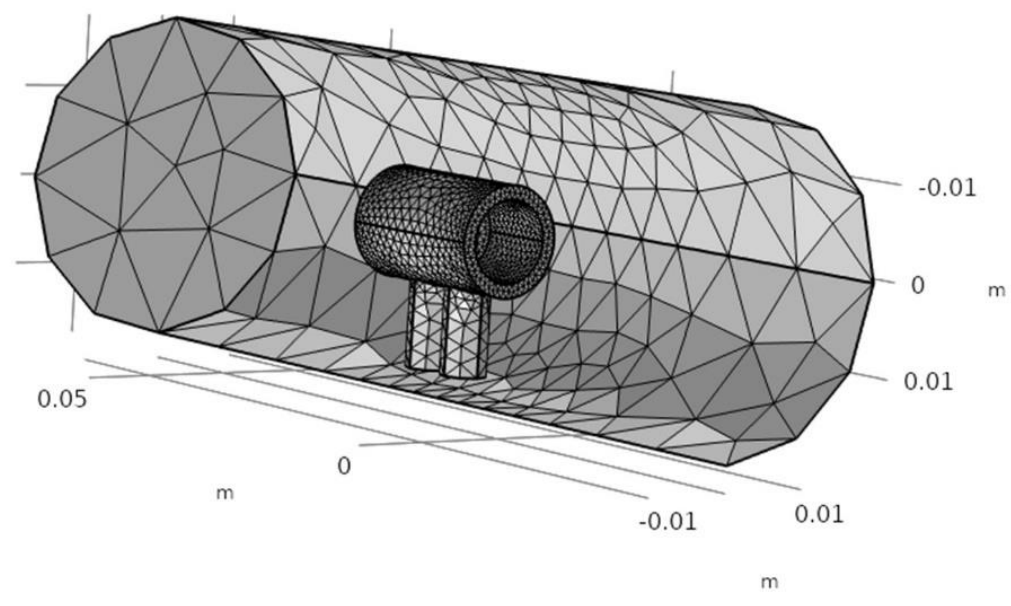

Figure A5: Scheme of the 3D meshed model showing the bidirectional probe inside a turbulent flow. 
Several 2D views are presented in figure A6 relative to dynamic pressure and velocity according to the angle of deviation of the probe. The mean flow velocity is $15 \mathrm{~m} / \mathrm{s}$. This can be used to obtain the difference of pressure between the two sides of the probe. The red curve of figure A4 represents this difference. It appears that the model provides a very good prediction of the influence of the angle effect up to $30 / 40^{\circ}$.
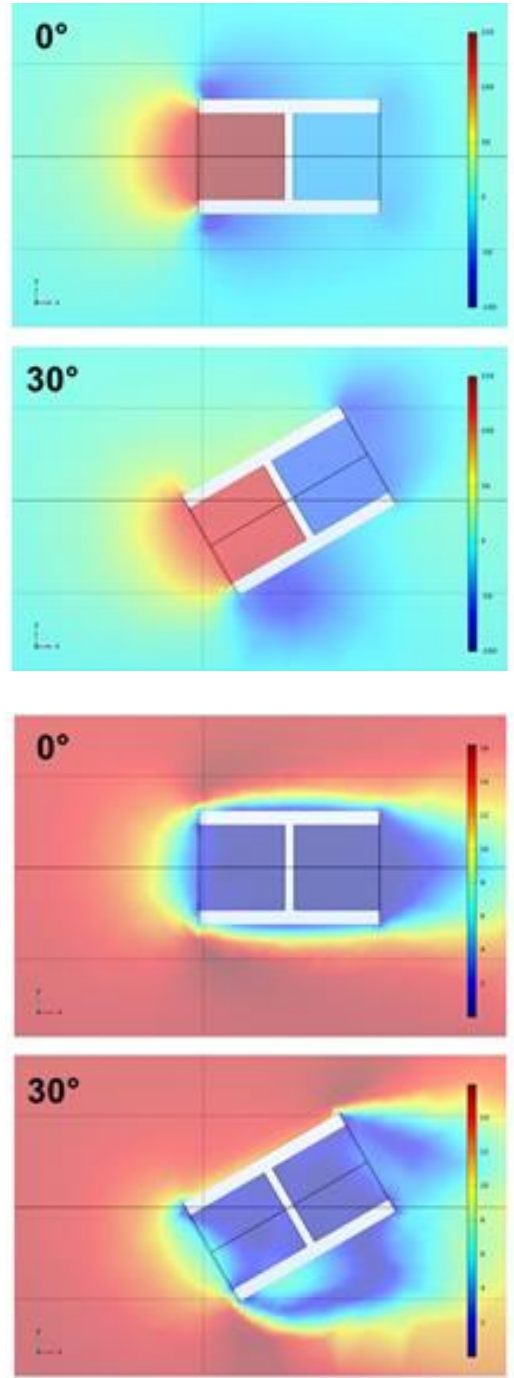
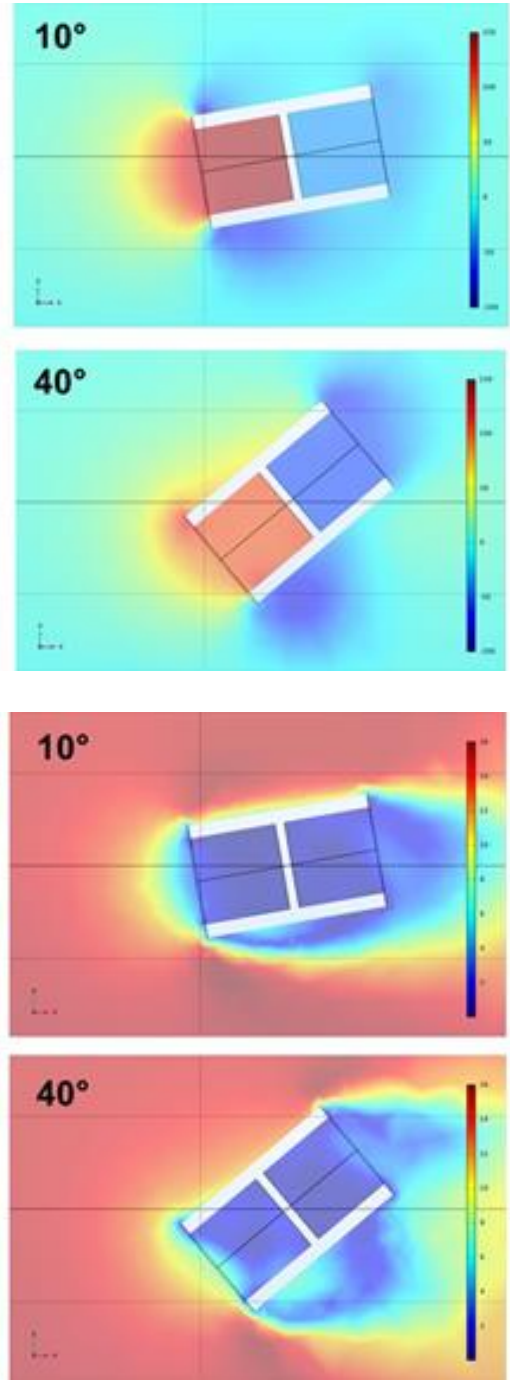
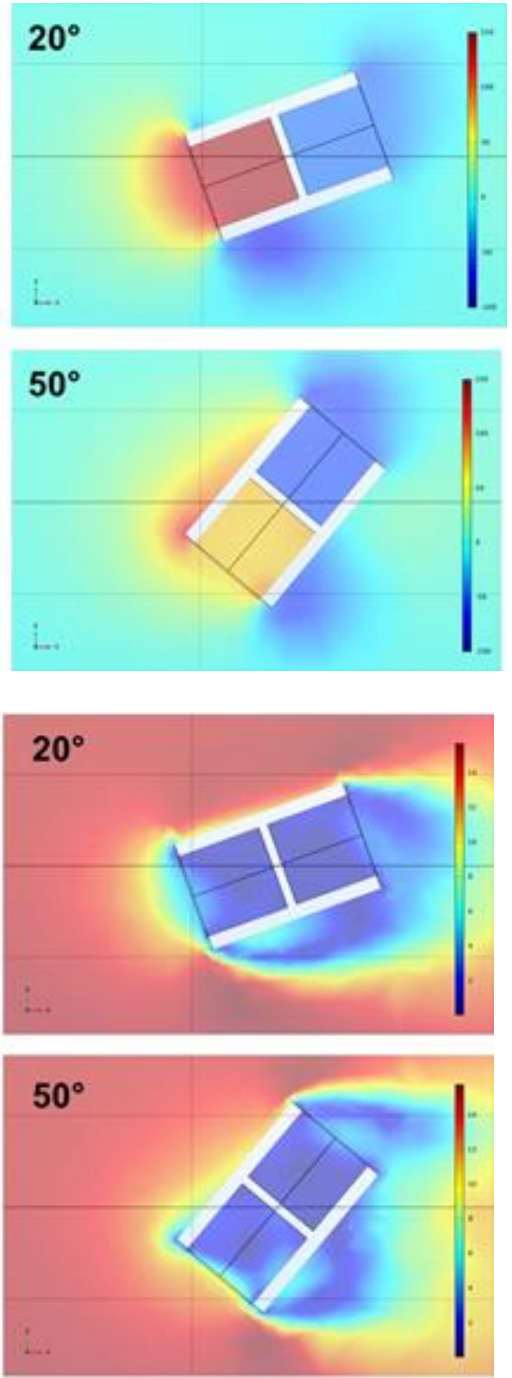

Figure A6: COMSOL model for the bidirectional probe. Influence of the angle of the head with the direction of the flow. Dynamic pressure (upper views, range: $-100 /+150 \mathrm{~Pa}$ ) and velocity (bottom views, range: $0 / 16 \mathrm{~m} / \mathrm{s}$ ).

Figure A7 shows the pressure calculated in the two cavities according to the angle of deviation. The differential pressure decreases rapidly from the angle of $30^{\circ}$ since the pressure in the cavity facing the flow drops while the pressure in the rear cavity remains stable. The model probably does not evaluate correctly the aeraulic of a tilted pipe mouth subjected to a local swirl. 


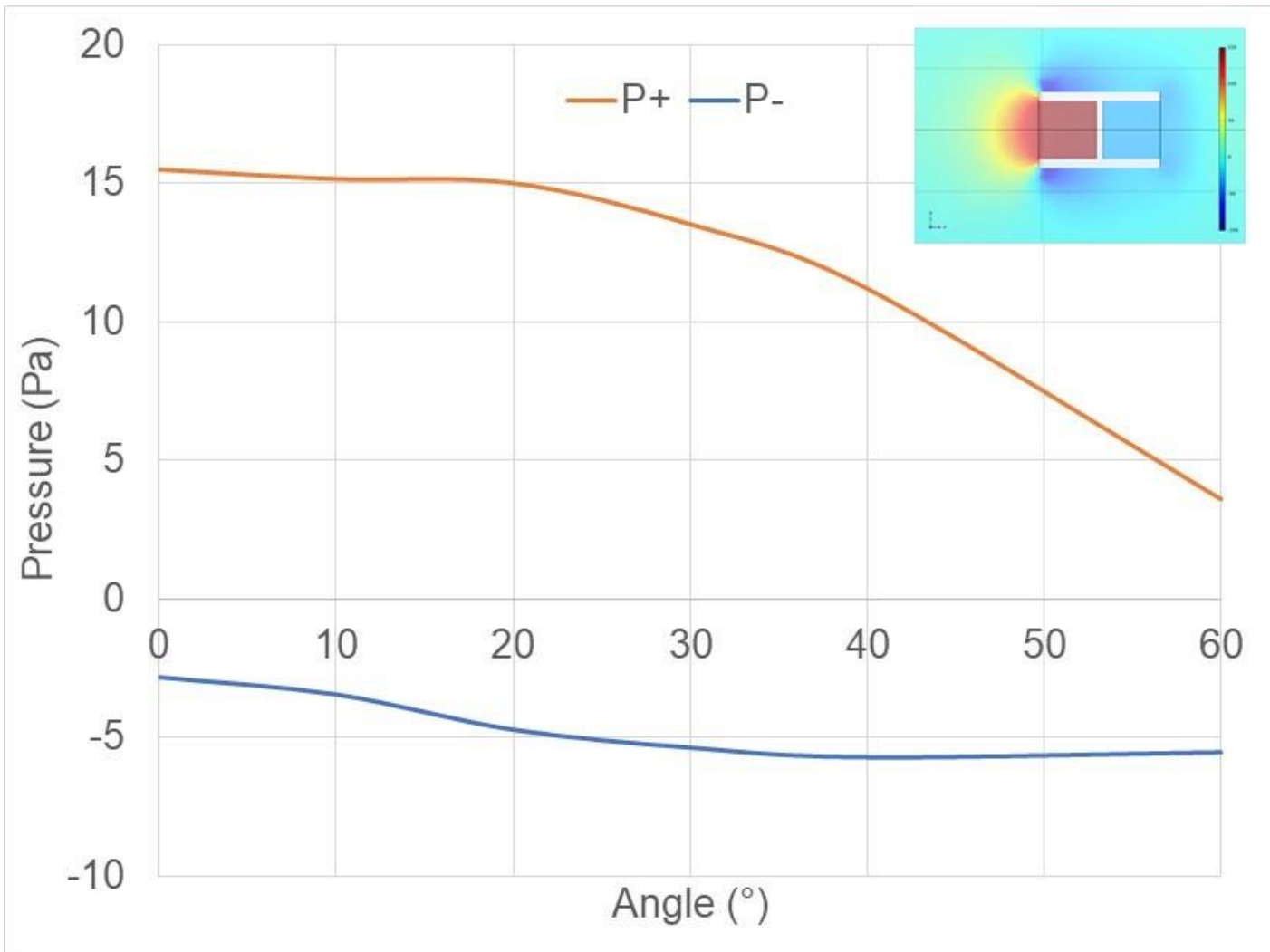

Figure A7: COMSOL model prediction of upstream and downstream pressure inside the cavities of the probe according to the angle toward the direction of the flow

\section{References}

LIU C. Y., WONG Y. W., CHAN W. K., GAN T. C. (1990), "Note on the robust bi-directional low velocity probe" - Experiments in fluids 9 (1990) pp354-356

SETTE B. J. G. (2005), "Critical considerations on the use of a bi-directional probe in heat release measurements » - Fire Mater. 2005; vol 29 ; pp 335-349

YUN B. J., EUH D. J., KANG K. H., SONG C. H., BAEK W. P. (2005), "Measurement of the single- and two-phase flow using a newly developed average bi-directional flow tube" Nuclear Engineering and technology, vol37, n6 December 2005 
Appendix B: data reduction of turbulence signals

Figure B1-a shows a typical pressure record obtained using a bidirectional probe (medium head, pipe length of $100 \mathrm{~mm}$ ) located on the axis of the air jet, at $\mathrm{x}=50 \mathrm{~cm}$ from the $5 \mathrm{~mm}$ diameter orifice (stagnation pressure $=642 \mathrm{mbar}$ ). Data were recorded at a rate of $5 \mathrm{kHz}$ over a period of 6 seconds to optimize the subsequent data reduction process as explained below. Note the sampling frequency is chosen about five times larger than the cut off frequency of the transducer.

Note that the range of the differential pressure sensor must be chosen according to the expected velocity fluctuations due to the turbulence. It was observed that the range should be threefold that corresponding to the average velocity of the flow. For instance, if the average flow velocity is $15 \mathrm{~m} / \mathrm{s}$, corresponding to a dynamic pressure of $200 \mathrm{~Pa}$, a minimum range of 0-600 Pa should be chosen.

A velocity time signal (Figure B1-b) is extracted from the pressure data using the correlation show in appendix A. Then a time average velocity is calculated is the standard way. It can be useful to subtract this mean value from the raw signal to obtain the velocity fluctuations (Figure B1-c). The latter should be zero-centred if the flow conditions are steady. The standard deviation of these fluctuations ( $u^{\prime}$ ) should be constant with time too. The turbulence intensity $\mathrm{u}$ '/U is then deduced.

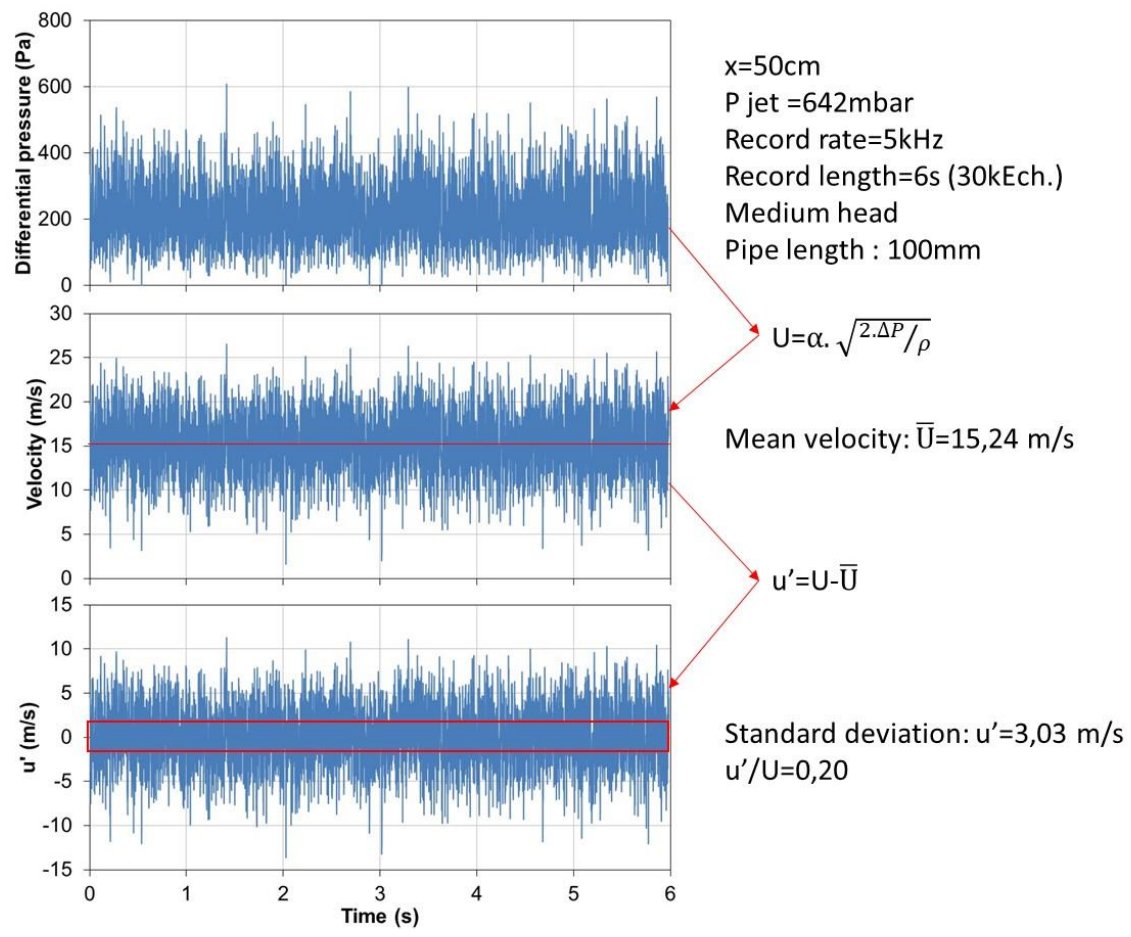

Figure B1: Analysis of the raw signal (medium head-100 $\mathrm{mm}$ connecting pipe, on the jet axis at $50 \mathrm{~cm}$ from the orifice under a 642 mbar static pressure)

The integral scale of the turbulence, $\mathrm{L}_{\mathrm{int}}$, can be deduced from this time signal as follows.

First, a discrete autocorrelation process is applied over at least a part $\left(\mathrm{t}_{\text {meas }}\right)$ of the velocity fluctuation signal [b1]. The integral autocorrelation time $\mathrm{L}_{t}$ is calculated over a time slot $\mathrm{t}_{\text {lim }}$ possibly shorter than $t_{\text {meas. }} \Delta t$ is the time step of the signal.

$$
R(\tau)=\sum_{t=0}^{t_{\text {meas }}} \frac{u^{\prime}(t) \cdot u^{\prime}(t-\tau)}{u^{\prime 2}} \Delta t
$$


By definition, $\mathrm{L}_{t}$ corresponds to the hatched area under the autocorrelation curve (figure B2-a). Under the Taylor assumption, the space integral scale $\mathrm{L}_{\text {int }}$ is (roughly) estimated using $\mathrm{L}_{\mathrm{t}} \bar{U}$. It may be difficult to assign a value to $t_{\text {lim }}$ when applying equation [b2] since oscillations may be superimposed (as shown on this example) over the expected $\mathrm{R}(\mathrm{t})$ curve (exponential law b3). Fitting the experimental curve with relation [b3] to obtain $\mathrm{L}_{\text {int }}$ may be a solution issue as indicated on figure B2-b.

$R=e^{\left(-x / L_{i n t}\right)}$
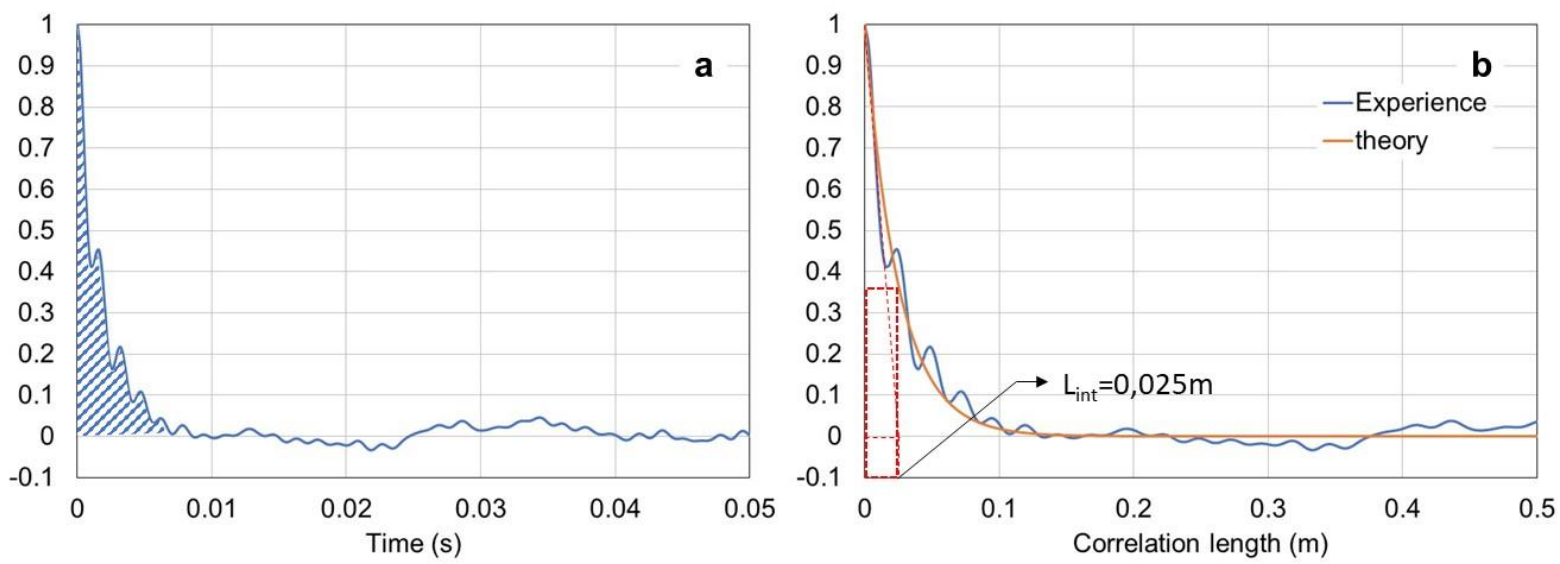

Figure B2: correlation curves and estimation of $L_{i n t}$

In this example, the correlation seems lost after $0.01 \mathrm{~s}$, so the integration times, $\mathrm{t}_{\text {lim }}$ and $\mathrm{t}_{\mathrm{meas}}$, do not need to be as long as the recorded duration (here 6 seconds). Since the average and integral values are not expected to change over the recording period ( $6 \mathrm{~s}$. here), the signal analysis [b1] and [b2] can be done on several parts of period and should provide the same results. It is not exactly so, not because of variations in the flow but because of other disturbances. It can then be justified to average several autocorrelation curves to smooth out the disturbances. To do this, the Bartlett method (Bartlett M. S. 1948) can be implemented. The record is divided in $n$ successive slots of duration $\delta, \delta$ being several times larger than Lt (figure B3-a). The autocorrelation curves are then calculated on each slot and an averaged final autocorrelation curve is calculated (figure B3 b). As shown later, the averaged autocorrelation curve provides much more accurate energy spectra. In figure B3-b, the influence of $\delta$ is shown. The averaged autocorrelation curves seem to merge when $\delta$ is larger than 0.34-0.6 s. A clearer indication is found in figure B3-c where $\mathrm{L}_{\text {int }}$ is extracted from the averaged autocorrelation curve. A correct value is obtained when $\delta$ is larger than about $0.5 \mathrm{~s}$. 

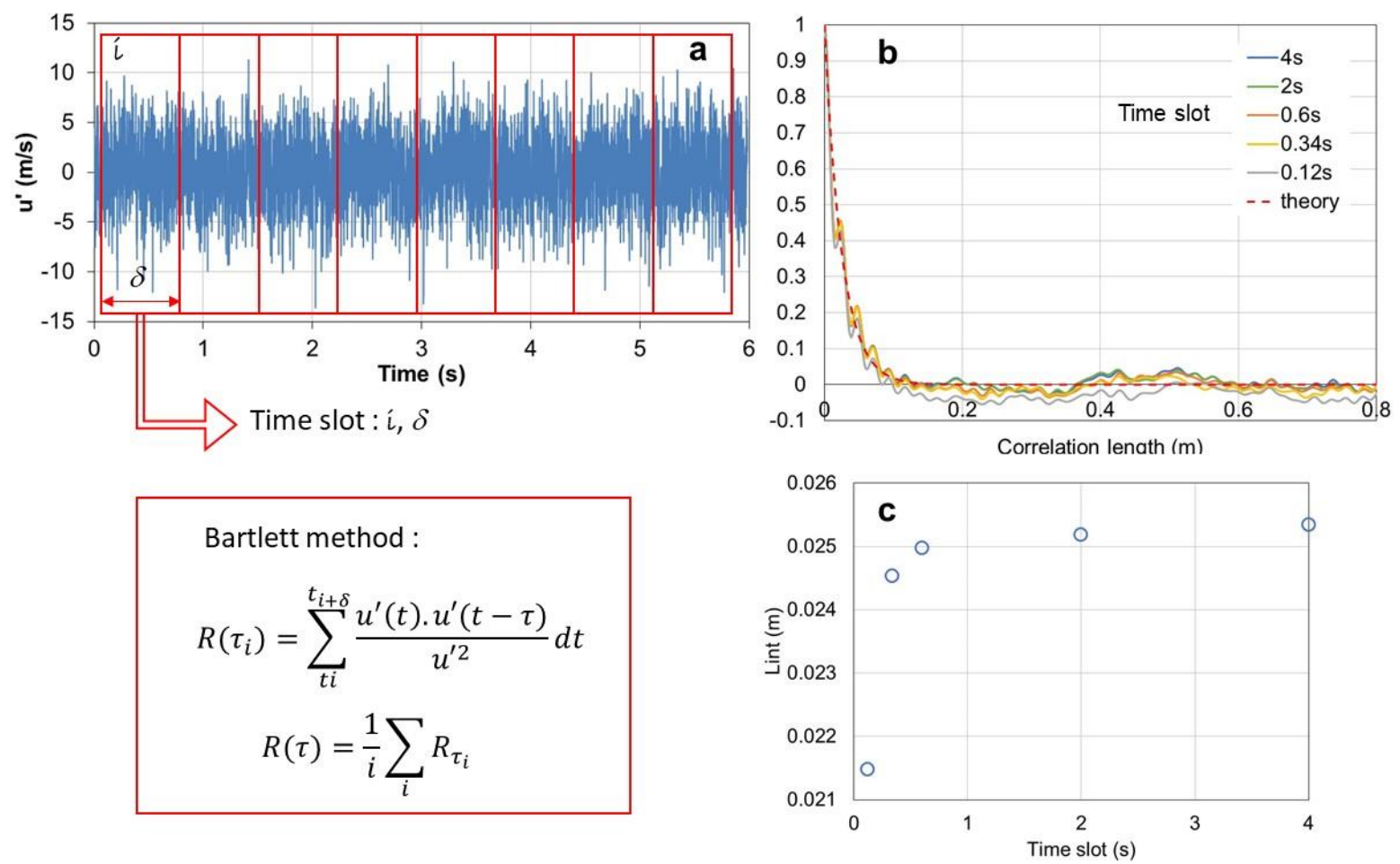

Figure B3: method to obtain the correlation curve from the raw signal (from figure B1-c)

To obtain the energy spectrum, a Fourier transform of the space correlation curve is done. A direct Fourier transform technique (expression b5) was selected for reasons to be explained below. The impact of the number of slots is shown on figure B4. The larger the number of slots the better the accuracy. But when the duration of the slot is too short (below $0.6 \mathrm{~s}$.), a significant drop in the large wavelengths (short wavenumbers) is observed because the contribution of the largest eddies was cut out.
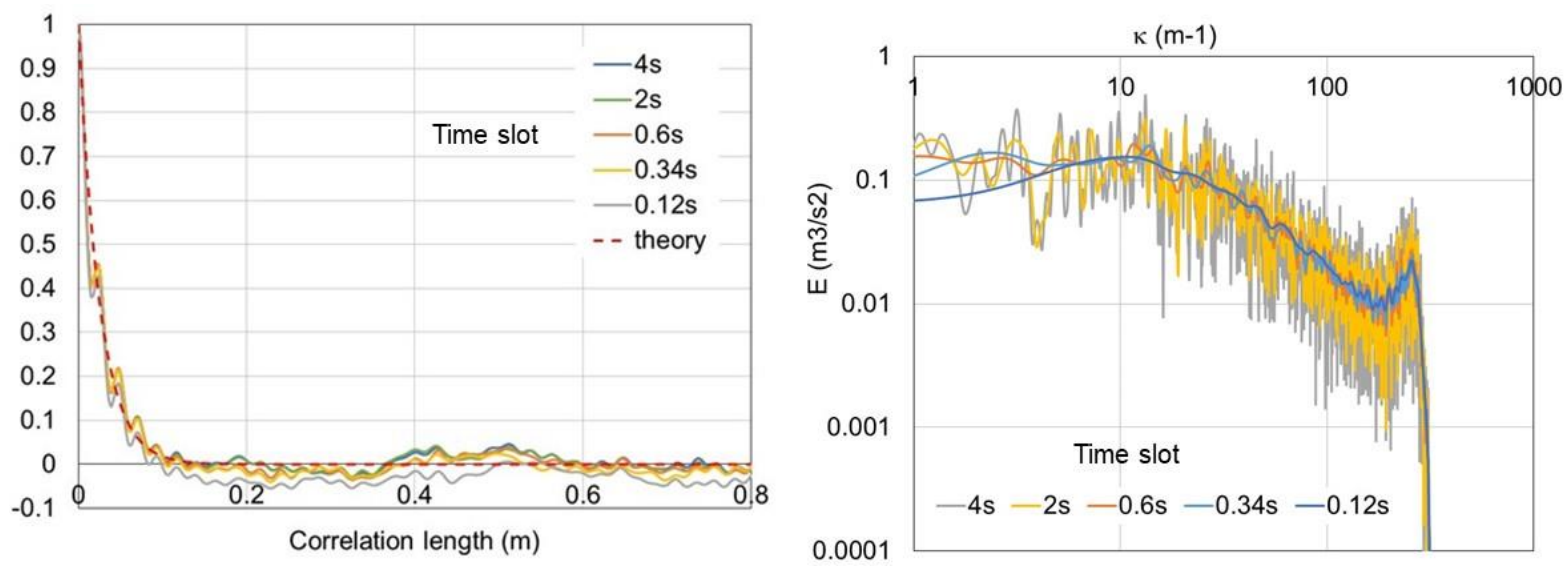

Figure B4: influence of the size/number of slots on the energy spectrum.

The Fourier transforms to obtain the spectrum could either be a direct transform (DFT), which is an integral (expression [b4]), or a fast Fourier transform (FFT) which is more a discrete version (expression [b5]). Is there a difference? 
$E(\kappa)=D F T(R)=\frac{2}{\pi} \cdot u^{\prime 2} \cdot \int_{0}^{\infty} R(x) \cdot \cos (\kappa \cdot x) \cdot d x$

$E(\kappa)=F F T(R)$

The results are presented on figure B5 and can be compared to the theoretical spectrum (expression [b6]) obtained using expression b3:

$E(\kappa)=\frac{2}{\pi} \cdot u^{\prime 2} \cdot L_{i n t} \cdot \frac{1}{1+\kappa^{2} \cdot L_{\text {int }}^{2}}$

The three spectra compare well but the DFT seems closer to the theoretical curve. This may come from the fact that the FFT is based on local values of the correlation curve and even though the Bartlett method smoothes out the disturbances, some remain and cover all the curve. This would add artificially "energy" on many points so that the calculated FFT spectrum should be somewhat overestimated. On the contrary, DFT adds an additional smoothing (as an integral) along the correlation curve so that most disturbances are destroyed. The fitting with the theoretical curve is then better.

Lastly, the methodology presented above, which fits closely to the definitions of the theory of turbulence, is not always applied. The method is tedious and short cuts may be used. One could note that the correlation signal is the convolution of $u$ ' $(t)$ with itself. The Fourier transform (FT) of this convolution, which is the energy spectrum, is equal to the square of the Fourier transform of $u^{\prime}(t)$ (expression [b7]).

$E(\kappa)=F T(R)=[F T(S)]^{2}$

This estimate is shown on figure B5 when the chosen FT is a DFT. If the use of [b7] provides a reasonable estimate of the energy spectrum, it is very inaccurate. Certainly, the various smoothing effects provided by the Bartlett method play an important role.

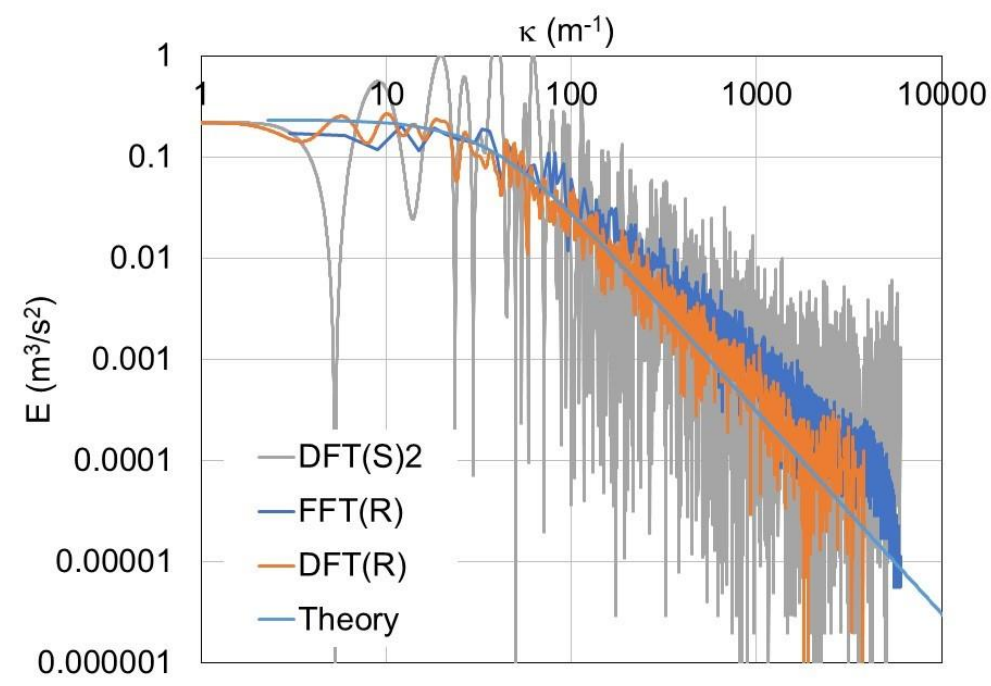

Figure B5: Various methods to derive the turbulence energy spectrum 
Appendix C: influence of the cut-off distance of the probe on the measured turbulence

If the probe could capture infinitesimal swirl, so infinite wavenumbers, the exact level of turbulence will be obtained since:

$$
\int_{0}^{+\infty} E(k) \cdot d k=u^{\prime 2}
$$

But all transducers are subject to limitations and specifically to frequency limitations. If $\mathrm{f}_{\mathrm{c}}$ is the cut-off frequency of the device, it may be associated to a cut off length scale and cut-off wave number as:

$$
L_{\text {cut }- \text { off }}=\frac{U}{f_{c}} \quad \Lambda_{\text {cut }- \text { off }}=\frac{2 \cdot \pi}{L_{\text {cut }- \text { off }}}
$$

So the transducer can only integrate the part of the spectrum between 0 and $\Lambda_{\text {cut-off }}$ wavenumbers and the resulting measured turbulence is:

$$
u_{\text {meas }}^{\prime 2}=\int_{0}^{\Lambda_{\text {cut }- \text { off }}} E(k) \cdot d k=u^{\prime 2} \cdot\left[\frac{2}{\pi} \cdot \tan ^{-1}\left(\Lambda \cdot L_{\text {int }}\right)\right]_{0}^{\Lambda_{\text {cut }-o f f \cdot L_{\text {int }}}}
$$

Setting $\Lambda_{\text {int }}=\frac{2 \cdot \pi}{L_{\text {int }}}$, a graphical representation of the ration $\mathrm{u}^{\prime}$ meas $/ \mathrm{u}$ ' as function of the cut-off is shown on figure $\mathrm{C} 1$. An underestimation of $\mathrm{u}^{\prime}$ meas of $1 \%$ is then expected for a cut-off frequency 5 time greater than the frequency associated with Lint. If the cut-off frequency is of the same order of magnitude than Lint frequency, an under estimation of $5 \%$ if expected.

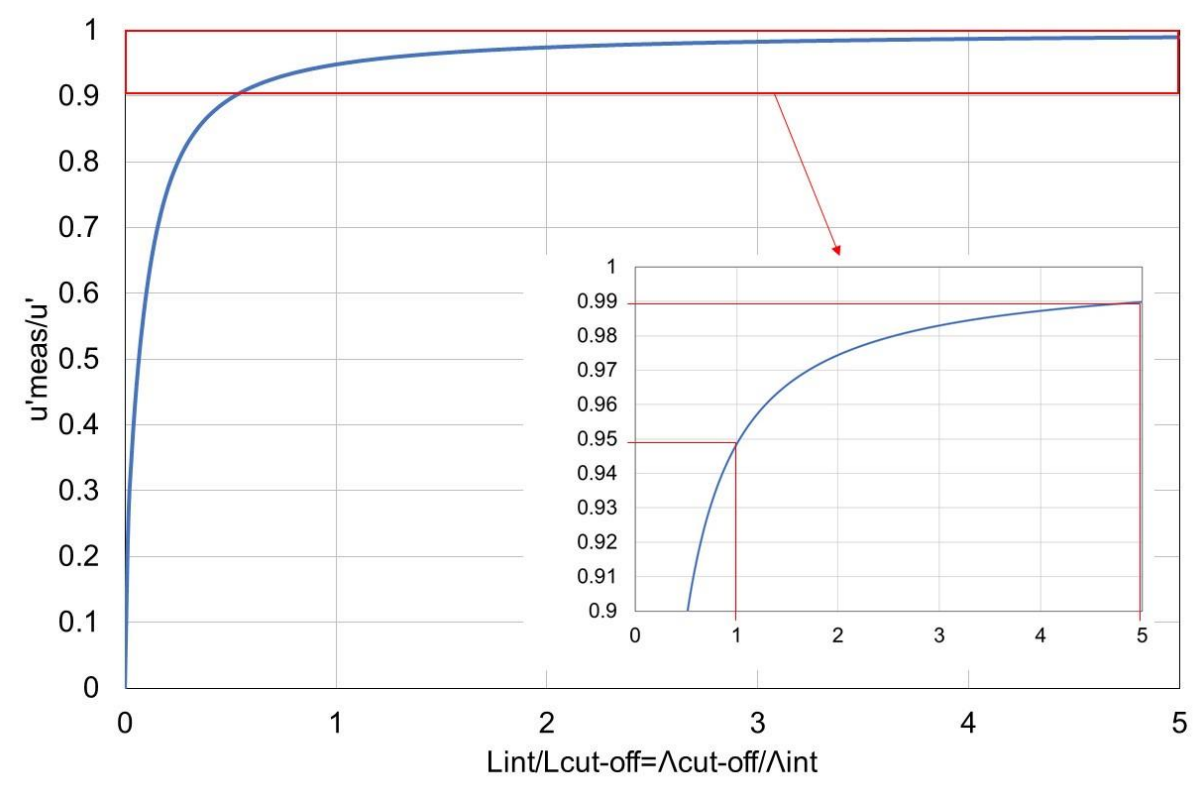

Figure C1: accuracy of the measurement of the turbulence as function of the cut-off limit of the probe 
Appendix D : mechanical oscillations of the sensor

In modelling the mechanical oscillations of the sensor, the piping are considered as beams and the head as a load. The pipes are supposed to be rigidly anchored at the differential pressure location. Mechanically speaking this arrangement can be modelled as a portal in which the transverse beam represents the head of the sensor. This "beam" is attached rigidly to the other "beams" (Figure D1).
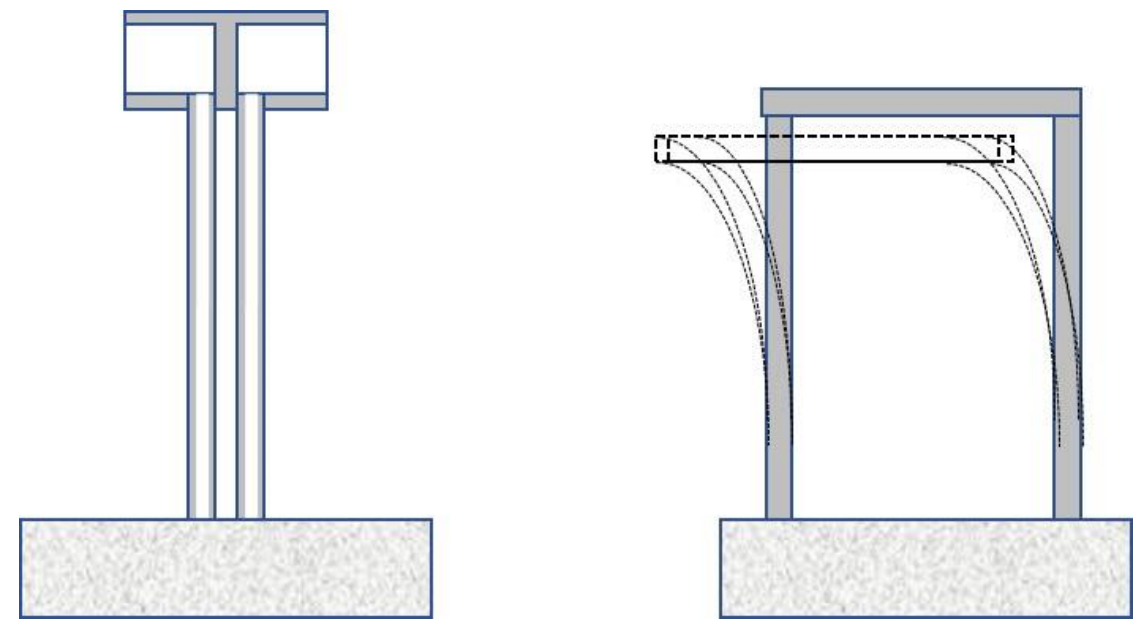

Figure D1: mechanical modelling of the sensor

This is a typical spring mass system in which the stiffness of the spring is due to the elastic flexion of the vertical beams. All beams are cylindrical tubes. The mathematical formulation is standard and can be found in any textbook.

The pulsation of the fundamental mode of vibration of a spring mass system is:

$$
\omega_{c}=\sqrt{\frac{K}{M}}
$$

Where $\mathrm{K}$ is the stiffness of the spring and $\mathrm{M}$ the mass which is oscillating. Applied to the rigid portal of figure $\mathrm{D} 1, \mathrm{~K}$ is given as:

$$
K=\frac{12 \cdot E \cdot(I+I)}{L^{3}}
$$

Where $\mathrm{E}$ is the Young modulus of the material (steel here), I the quadratic moment of flexion of a vertical beam. This moment $\mathrm{I}$ and $\mathrm{M}$ are:

$$
M \cong \rho_{\text {steel }} \cdot\left[\frac{\pi}{4} \cdot D_{\text {head }}{ }^{2} \cdot e+\pi \cdot D_{\text {head }} \cdot e \cdot L_{\text {head }}\right]+\frac{5}{12} \cdot 2 \cdot \rho_{\text {steel }} \cdot\left[\pi \cdot D_{\text {pipe }} \cdot e \cdot L_{\text {pipe }}\right]
$$




$$
I=\frac{\pi}{64} \cdot\left(D_{\text {head }}^{4}-\left(D_{\text {head }}-e\right)^{4}\right)
$$

Where $\mathrm{D}_{\text {head }}$ and $\mathrm{L}_{\text {head }}$ are respectively the external diameter and the total length of the head of the sensor, e the thickness of the metal $\left(\rho_{\text {steel }}\right.$ is the specific mass of the material $=$ steel). $D_{\text {pipe }}$ and $\mathrm{L}_{\text {pipe }}$ are respectively the external diameter and the total length of the connecting pipes. The factor 5/12 accounts for the fact that the part of the pipe close to the anchoring point hardly move (so do not participate to the acceleration) and the factor 2 because there are two connecting pipes.

When applied to the sensor with $10 \mathrm{~cm}$ long connecting pipes (4 $\mathrm{mm}$ external diameter of the connecting pipes) and a medium head ( $D_{\text {head }}=10 \mathrm{~mm}$; $\left.\mathrm{L}_{\text {head }}=20 \mathrm{~mm} ; \mathrm{e}=1 \mathrm{~mm}\right)$, the frequency of the oscillation is about $600 \mathrm{Kz}$. With $60 \mathrm{~cm}$ long connecting pipes, this frequency drops to 10 $\mathrm{Hz}$. With the same connecting pipes, small heads ( $\mathrm{D}_{\text {head }}=5 \mathrm{~mm}$; $\mathrm{L}_{\text {head }}=10 \mathrm{~mm} ; \mathrm{e}=1 \mathrm{~mm}$ ) oscillate at higher frequencies (respectively $750 \mathrm{~Hz}$ and $15 \mathrm{~Hz}$ ).

This falls into the frequency ranges where "bumps" into the turbulence spectra where detected.

But the dependency of the characteristic frequency of the bumps to the length of the connecting pipe (linear) is not as pronounced as found with this mechanical modelling (cubic dependency). But more important perhaps, given the aerodynamic forces applied on the head of the sensor the maximum deflection (medium head in a $15 \mathrm{~m} / \mathrm{s}$ stream of air) is a micrometer and the related oscillation velocity is a $\mathrm{mm} / \mathrm{s}$ at most. This is much below the detection level of the sensor. 
Appendix E: Acoustic of the probe with its sensor

The assembly of the bidirectional probe with its differential pressure sensor is represented on figure E1-A.

Finding a general analytic solution to sound propagation in such a device is a difficult task. For instance, Ruud van Ommen et al (1999) proposed an analytic model giving accurate results when predicting resonance frequencies in long tube assemblies connected to cavities but at the cost of a significant complexity. In the present context it was decided to use a simplified model to check the acoustic assumption.
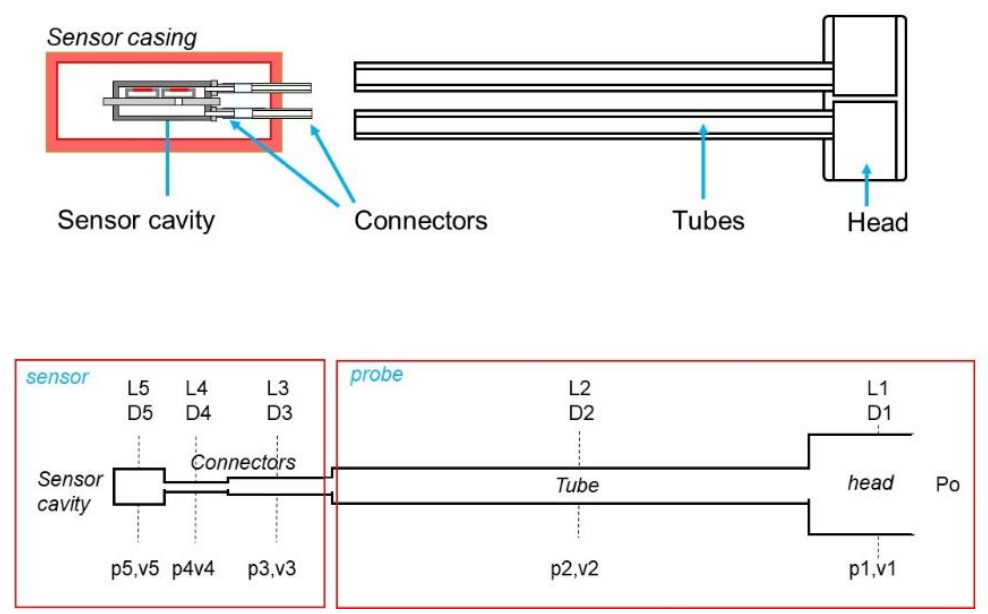

B

Figure E1. A: Schematic view of the turbulence probe and its differential pressure sensor. B: acoustic representation

Acoustically, figure E1-B is a model of the device (half of it in fact) as a series of interconnected tubes. Basic dimensions (length and diameter) are those of each modelled section of pipe. An impedance is affected to each tube which accounts for situations where the tube length can be larger than the wavelength using the concept of reduced impedance (Kinsler 1982, A. Chaigne 2003, V. Martin 2007). The pressure $P_{i}$ and velocity $V_{i}$ quantities at one extremity (labelled 0 ) of a pipe may be evaluated from the values at the opposite extremity (labelled 1) as:

$\left\{\begin{array}{l}P_{0} \\ V_{0}\end{array}\right\}=\left[\begin{array}{cc}\cos (\kappa L) & i \rho c \sin (\kappa L) \\ -\frac{i}{\rho c} \sin (\kappa L) & \cos (\kappa L)\end{array}\right]\left\{\begin{array}{l}P_{1} \\ V_{1}\end{array}\right\}$

With:

- $\quad \kappa:$ wave number

- $\quad \rho:$ mean air density

- c: sound speed

- L: tube length

A pressure perturbation is assumed in the transducer cavity $(\mathrm{V} 1=0)$. Pressures and velocities are calculated incrementally throughout the series of impedances (e.g. tubes) up the inlet of the 
head under the further assumption that acoustic flowrate is preserved at each cross-section change:

$$
V_{n-1}=V_{n}\left(S_{n} S_{n-1}\right)
$$

This model was used to calculate the resonance frequencies of the various probe geometries used during the experiments (For each geometry $\kappa$ was varied until the resonance was found). The results are presented in Table E-I with experimental resonance frequencies. A graphical presentation is given in figure E-2.

Table E-I Probe dimensions, experimental and calculated resonance frequencies

\begin{tabular}{|c|c|c|c|c|c|c|}
\hline & \multicolumn{2}{|l|}{ Head } & \multicolumn{2}{|l|}{ Tubes } & \multirow{2}{*}{\begin{tabular}{|c} 
measured \\
frequency \\
$(\mathrm{Hz})$ \\
\end{tabular}} & \multirow{2}{*}{\begin{tabular}{|c} 
calculated \\
frequency \\
$(\mathrm{Hz})$
\end{tabular}} \\
\hline & $\begin{array}{l}\text { length } \\
(\mathrm{mm})\end{array}$ & $\begin{array}{c}\text { diameter } \\
(\mathrm{mm})\end{array}$ & $\begin{array}{c}\text { diameter } \\
(\mathrm{mm})\end{array}$ & $\begin{array}{c}\text { length } \\
(\mathrm{mm})\end{array}$ & & \\
\hline \multirow{11}{*}{$\begin{array}{c}\text { medium } \\
\text { head }\end{array}$} & \multirow{11}{*}{9.5} & \multirow{11}{*}{10} & 3 & 55 & 715 & 674 \\
\hline & & & 2.6 & 60 & 548 & 636 \\
\hline & & & 2.6 & 60 & 572 & 636 \\
\hline & & & 3.5 & 75 & 630 & 653 \\
\hline & & & 3.5 & 75 & 674 & 653 \\
\hline & & & 3 & 140 & 543 & 480 \\
\hline & & & 3 & 180 & 524 & 401 \\
\hline & & & 3 & 190 & 340 & 384 \\
\hline & & & 3 & 350 & 215 & 226 \\
\hline & & & 3 & 600 & 126 & 137 \\
\hline & & & 3 & 600 & 123 & 137 \\
\hline \multirow{9}{*}{$\begin{array}{l}\text { small } \\
\text { head }\end{array}$} & \multirow{9}{*}{5} & \multirow{9}{*}{4.8} & 1.2 & 50 & 552 & 486 \\
\hline & & & 1.2 & 60 & 429 & 456 \\
\hline & & & 1.2 & 100 & 296 & 387 \\
\hline & & & 1.2 & 100 & 332 & 308 \\
\hline & & & 1.2 & 150 & 292 & 299 \\
\hline & & & 1 & 150 & 222 & 299 \\
\hline & & & 1.2 & 185 & 180 & 265 \\
\hline & & & 1.2 & 600 & 171 & 131 \\
\hline & & & 1.2 & 600 & 195 & 131 \\
\hline \multirow{2}{*}{ Big head } & \multirow{2}{*}{24} & \multirow{2}{*}{22.5} & 4 & 130 & 598 & 543 \\
\hline & & & 4 & 130 & 577 & 543 \\
\hline
\end{tabular}




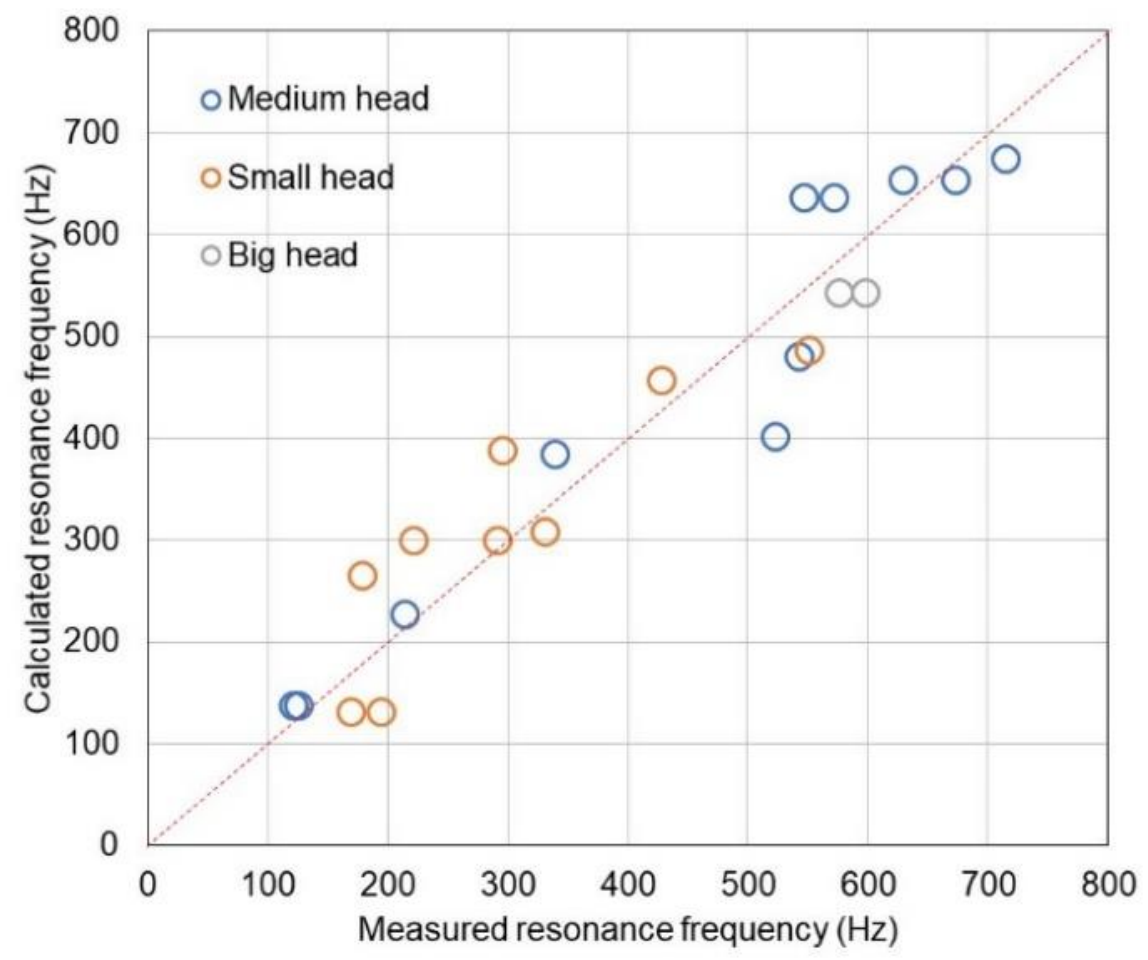

Figure E-2: Experimental resonance frequencies versus calculated ones

Calculated and measured resonance frequencies correlate well. Scattering may result from geometrical approximations made to establish the model shown in figure E1-B (unideal junctions between the tubes, for instance). Nevertheless, this proves the internal acoustics is responsible for the "bumps" on the turbulence spectra.

This simple model may help to optimize the geometry of the probe. Internal dimensions of the pressure transducer are fixed and cannot be changed (given in table E-II). It was verified that the scattering in the resonance frequency due to uncertainty about the dimensions of the transducer cavity is very small (less than $5 \%$ ).

Table E-II Pressure sensor relevant dimensions

\begin{tabular}{lll}
\hline Sensor dimension & Length $(\mathbf{m m})$ & Diameter $(\mathrm{mm})$ \\
\hline Sensor Cavity & $8+/-0.5$ & $4.5+/-0.5$ \\
Sensor connector & $5+/-0.5$ & $0.8+/-0.1$ \\
Casing connector & $13+/-0.5$ & $1.4+/-0.1$ \\
\hline
\end{tabular}

If was further observed experimentally that in most situation the resonance was followed by the cut off of the turbulence signal. Thus, to obtain accurate enough turbulence measurement under the experimental conditions covered in this paper $\left(15 \mathrm{~m} / \mathrm{s}\right.$ at $50 \mathrm{~cm}$, so $\mathrm{L}_{\text {int }} \sim 2.5 \mathrm{~cm}$ and $\mathrm{f}_{\text {int }} \sim$ $600 \mathrm{~Hz}$ ), the cutoff frequency (resonance frequency) of the probe should not be smaller than $600 \mathrm{~Hz}$ (to cover the jets turbulent scale). And the higher the resonance frequency, the better the turbulence measurements. It appears that the medium head with the shorter tube length $(55 \mathrm{~mm})$ 
represents the best solution (with a calculated resonance frequency of $675 \mathrm{~Hz}$ and a measured one of $715 \mathrm{~Hz}$ ).

To increase even more the resonance frequency while preserving a minimum length of $50 \mathrm{~mm}$ for practical reasons (handling on experimental site), the model suggests using larger tube diameter and slightly larger probe head.

Of course, experimental constraints may need to adapt the design of the probes (confined spaces, high jet momentum,..) and, knowing the turbulence range to measure, the proposed model may help to optimize the design.

CHAIGNE A. (2002), “Ondes acoustiques" - Ecole Polytechnique Eds

KINSLER L.E., FREY, A.R., COPPENS A.B., SANDERS J.V. - Fundamentals of acoustics. John Wiley \& Sons, 3e ed. (1982)

MARTIN V. (2007), "Elements d'Acoustique Générale" - EPFL Press

RUUD VAN OMMEN J., SCHOUTEN J. C., VANDER STAPPEN M. L. M., VAN DEN BLEEK C. M. (1999) "Response characteristics of probe - transducer systems for pressure measurements in gas - solid fluidized beds: how to prevent pitfalls in dynamic pressure measurements" - Powder Technology - 106 (1999) 199-218 